\title{
Toward exotic 6D supergravities
}

\author{
Yannick Bertrand $\odot,{ }^{1, *}$ Stefan Hohenegger $\odot,{ }^{1, \dagger}$ Olaf Hohm, ${ }^{2, *}$ and Henning Samtleben $\odot^{3, \S}$ \\ ${ }^{1}$ Univ Lyon, Univ Claude Bernard Lyon 1, CNRS/IN2P3, IP2I Lyon, UMR 5822, \\ F-69622, Villeurbanne, France \\ ${ }^{2}$ Institute for Physics, Humboldt University Berlin, Zum Großen Windkanal 6, D-12489 Berlin, Germany \\ ${ }^{3}$ Univ Lyon, Ens de Lyon, Univ Claude Bernard, CNRS, Laboratoire de Physique, F-69342 Lyon, France
}

(Received 14 October 2020; accepted 8 December 2020; published 3 February 2021)

\begin{abstract}
We investigate exotic supergravity theories in 6D with maximal $(4,0)$ and $(3,1)$ supersymmetry, which were conjectured by Hull to exist and to describe strong coupling limits of $\mathcal{N}=8$ theories in 5D. These theories involve exotic gauge fields with nonstandard Young tableaux representations, subject to (self-) duality constraints. We give novel actions in a $5+1$ split of coordinates whose field equations reproduce those of the free bosonic $(4,0)$ and $(3,1)$ theory, respectively, including the (self-)duality relations. Evidence is presented for a master exceptional field theory formulation with an extended section constraint that, depending on the solution, produces the $(4,0),(3,1)$ or the conventional $(2,2)$ theory. We comment on the possible construction of a fully nonlinear master exceptional field theory.
\end{abstract}

DOI: $10.1103 /$ PhysRevD.103.046002

\section{INTRODUCTION}

Among the surprising features of string/M-theory is the possible existence of exotic superconformal field and gravity theories in six dimensions, which display generalizations of electric-magnetic duality. Specifically, the supermultiplets of these theories are such that the corresponding fields must be subject to (self-)duality constraints, some of which involve exotic Young tableaux representations. In this paper our focus will be on a conjecture by Hull $[1,2]$ according to which there are strong coupling limits of $\mathcal{N}=8$ theories in five dimensions that are given by six-dimensional theories with chiral $\mathcal{N}=(4,0)$ and $\mathcal{N}=$ $(3,1)$ supersymmetry, respectively. Such theories must be exotic or nongeometric since they feature mixed symmetry tensors of Young tableaux type $\square$ and $\square$, respectively, instead of a conventional graviton, hence suggesting the need for a generalized notion of spacetime and diffeomorphism invariance. They are set to play a distinguished role among the maximally supersymmetric theories [3-5].

A possible window into these somewhat mysterious structures is offered by a Kaluza-Klein perspective from five dimensions. The supermultiplets of five-dimensional

\footnotetext{
*y.bertrand@ipnl.in2p3.fr

s.hohenegger@ipnl.in2p3.fr

*ohohm@physik.hu-berlin.de

shenning.samtleben@ens-lyon.fr
}

Published by the American Physical Society under the terms of the Creative Commons Attribution 4.0 International license. Further distribution of this work must maintain attribution to the author(s) and the published article's title, journal citation, and DOI. Funded by SCOAP ${ }^{3}$.
(5D) theories with maximal supersymmetry (32 real supercharges) were classified by Strathdee [6] and further clarified by Hull in [7]. The 5D superalgebra reads

$\left\{Q_{\alpha}^{a}, Q_{\beta}^{b}\right\}=\Omega^{a b}\left(\Gamma^{\mu} C\right)_{\alpha \beta} P_{\mu}+C_{\alpha \beta}\left(Z^{a b}+\Omega^{a b} K\right)$,

where $\alpha, \beta, \ldots=1, \ldots, 4$ are the space-time spinor indices and $a, b, \ldots=1, \ldots, 8$ are $\operatorname{USp}(8) R$-symmetry indices. This superalgebra features 27 central charges $Z^{a b}$, satisfying $Z^{a b}=-Z^{b a}, \Omega^{a b} Z_{a b}=0$, and a singlet central charge $K$. The short (or BPS after Bogomol'nyi-PrasadSommerfield) multiplets of this superalgebra describe the possible Kaluza-Klein towers that any six-dimensional (6D) theory with maximal supersymmetry displays when compactified on a circle. For the conventional maximal 6D supergravity, which features $\mathcal{N}=(2,2)$ supersymmetry, the massive Kaluza-Klein states do not carry the singlet central charge $K$. Instead, they carry a particular central charge $Z^{a b}$ transforming as a singlet under the six-dimensional $R$-symmetry group $\mathrm{USp}(4) \times \mathrm{USp}(4)$. In contrast, the massive multiplets of the exotic theories carry nonvanishing singlet charge $K$ [together with nonvanishing $Z^{a b}$, singlet under $\operatorname{USp}(4) \times \operatorname{USp}(4)$ in the case of the $\mathcal{N}=$ $(3,1)$ multiplets] [7]. This points to a unifying framework in the spirit of exceptional field theories [8-11] which we will elaborate on in this paper.

Exceptional field theory (ExFT) provides in particular a formulation of 11-dimensional (11D) and type IIB supergravity in a form that is covariant under the global symmetry group $\mathrm{E}_{6(6)}$ of $5 \mathrm{D}$ maximal supergravity, thanks to extended coordinates in the 27 representation of this group, which are added to the five coordinates of $5 \mathrm{D}$ 
supergravity. The resulting theory is thus based on a $(5+27)$-dimensional spacetime split, in which the $\mathbf{2 7}$ coordinates are subject to an $\mathrm{E}_{6(6)}$ covariant "section constraint" restricting them to a suitable physical subspace, from which the complete (untruncated) 11D supergravity can be reconstructed, albeit in a Kaluza-Klein type formulation with a $5+6$ split of coordinates. (Equivalently, one may think of this as coupling the infinite towers of massive Kaluza-Klein multiplets to 5D supergravity, which reconstructs the complete 11D supergravity.) A different physical section reproduces the IIB theory $[10,12]$. From a higherdimensional perspective, the extra coordinates can be thought of as accounting for the possible brane windings, which in turn are related to the 27 central charges of the supersymmetry algebra (1.1). The structure of the exotic supermultiplets then suggests an inclusion of their couplings within a suitable extension of this framework.

In this paper we will present actions for (the bosonic sectors of) the free exotic $6 \mathrm{D}$ theories that generalize the (linearized) $\mathrm{E}_{6(6)}$ exceptional field theory $[10,11]$ by adding one more "exotic" coordinate to the 27 , as suggested by the singlet central charge in (1.1). As one of the most enticing outcomes of our investigation we find evidence for a master exceptional field theory formulation in which the conventional $\mathcal{N}=(2,2)$ theory as well as the $\mathcal{N}=(4,0)$ and $\mathcal{N}=(3,1)$ theory are obtained through different solutions of an extended section constraint of the form

$d^{M N K} \partial_{N} \otimes \partial_{K}-\frac{1}{\sqrt{10}} \Delta^{M N}\left(\partial_{N} \otimes \partial_{\bullet}+\partial_{\bullet} \otimes \partial_{N}\right)=0$,

where $M, N=1, \ldots, 27$, are fundamental $E_{6(6)}$ indices, $d^{M N K}$ denotes the $E_{6(6)}$ invariant fully symmetric tensor, and $\partial$. is the derivative dual to the exotic coordinate. Moreover, $\Delta^{M N}$ denotes the (constant) background part of the generalized metric $\mathcal{M}^{M N}$ encoding all scalar fields. The first term in Eq. (1.2) defines the section constraint of $E_{6(6)}$ exceptional field theory, whose solutions restrict to the standard $D=11$ and IIB sections. The second term encodes the extension of the constraint allowing for two more solutions corresponding to $\mathcal{N}=(4,0)$ and $\mathcal{N}=(3,1)$, respectively. More precisely, we recover the $\mathcal{N}=(4,0)$ exotic theory by dropping all dependence on the 27 standard coordinates, and keeping only the dependence on the exotic coordinate. The $\mathcal{N}=(3,1)$ model in turn is recovered by superposing this coordinate with the $\mathrm{F}_{4(4)}$ singlet under $27 \rightarrow 26+1$ among the 27 coordinates of ExFT. While we give several independent pieces of evidence for the existence of this master formulation (some of which entail highly nontrivial numerical agreement), we also point out some gaps of the master formulation as understood so far. This implies that the complete nonlinear theory requires new ingredients, not the least of which is a section constraint that makes sense for the nonlinear theory and that reduces to (1.2) in the appropriate limit.

As a technical result, we present novel actions for the bosonic sectors of the $\mathcal{N}=(3,1)$ and the $\mathcal{N}=(4,0)$ model that are based on a $5+1$ split of the six-dimensional space-time, sacrificing manifest 6D Poincaré invariance. In the spirit of ExFT, these are two-derivative actions which upon dimensional reduction to five dimensions reduce to the same action of linearized maximal 5D supergravity. All dual fields, in particular the entire dual graviton sector, only appear under derivative along the sixth dimension. The full field equations obtained by variation combine the secondorder Fierz-Pauli equations with first-order duality equations defining the dual graviton sector. Actions for self-dual fields based on a $5+1$ split of spacetime date back to [13] with the description of self-dual $6 \mathrm{D}$ tensor fields. More recently, actions for the $\mathcal{N}=(3,1)$ and $\mathcal{N}=(4,0)$ models have been constructed in [14-16], based on the prepotential formalism developed in [17] in the context of linearized gravity. Introduction of prepotentials for the gauge fields adapted to their self-duality properties allows for the construction of an action of fourth order in spatial derivatives. Our construction is closer in spirit to the original construction of [13], albeit dual in a sense discussed in more detail in the Appendix A. It provides a novel mechanism for describing self-dual exotic tensor fields.

The rest of this paper is organized as follows. In Sec. II we review the bosonic sector of the $\mathcal{N}=(2,2), \mathcal{N}=(3,1)$ and $\mathcal{N}=(4,0)$ theories at the level of the equations motion, which are manifestly 6D Lorentz invariant. In order to find actions for these theories we abandon manifest Lorentz invariance by performing a $5+1$ split of coordinates in Sec. III for each of these models. In Sec. IV we then present, as one of our main technical results, actions whose secondorder Euler-Lagrange equations can be integrated in order to reproduce the correct dynamics of the three theories. In Sec. V we present the master formulation as currently understood, highlight its successes, which strike us as significant, but also discuss the structural problems that remain. We close with a brief outlook.

\section{REVIEW OF 6D MODELS}

We study six-dimensional field theories in Minkowski space with flat metric

$\eta_{\hat{\mu} \hat{\nu}}=\operatorname{diag}\{-1,1,1,1,1,1\}, \quad \hat{\mu}, \hat{\nu}=0, \ldots, 5$.

The Poincaré algebra in six dimensions admits chiral $\left(\mathcal{N}_{+}, \mathcal{N}_{-}\right)$supersymmetric extensions where $\mathcal{N}_{ \pm}$count the cumber of right- and left-handed supercharges, respectively [6]. In particular, maximal supersymmetry (32 real supercharges) allows for the three possibilities $\left(\mathcal{N}_{+}, \mathcal{N}_{-}\right)=(2,2),\left(\mathcal{N}_{+}, \mathcal{N}_{-}\right)=(3,1)$, and $\left(\mathcal{N}_{+}, \mathcal{N}_{-}\right)=$ $(4,0)$. The corresponding lowest-dimensional massless supermultiplets have the field content [6] 


$$
\begin{aligned}
(2,2): & (3,3 ; 1,1)+(2,2 ; 4,4)+(1,3 ; 5,1)+(3,1 ; 1,5) \\
& +(1,1 ; 5,5)+(2,3 ; 4,1)+(3,2 ; 1,4) \\
& +(2,1 ; 4,5)+(1,2 ; 5,4),
\end{aligned}
$$

$$
(3,1):(4,2 ; 1,1)+(2,2 ; 14,1)+(3,1 ; 6,2)+\left(1,1 ; 14^{\prime}, 2\right)
$$$$
+(4,1 ; 1,2)+(3,2 ; 6,1)+(2,1 ; 14,2)
$$$$
+\left(1,2 ; 14^{\prime}, 1\right) \text {, }
$$

$$
\begin{aligned}
(4,0): & (5,1 ; 1)+(3,1 ; 27)+(1,1 ; 42)+(4,1 ; 8) \\
& +(2,1 ; 48),
\end{aligned}
$$

organized into representations of the little group

$\mathrm{G}_{0}=\mathrm{SU}(2) \times \mathrm{SU}(2) \times \operatorname{USp}\left(2 \mathcal{N}_{+}\right) \times \operatorname{USp}\left(2 \mathcal{N}_{-}\right)$.

In this section, we briefly review the six-dimensional free theories associated to these multiplets.

\section{A. The $\mathcal{N}=(2,2)$ model}

Let us start from the $\mathcal{N}=(2,2)$ multiplet corresponding to maximal supergravity in six dimensions. Its bosonic field content comprises a metric, 25 scalar fields, 16 vectors, and 5 two-forms. The full nonlinear theory has been constructed in [18] with the scalar fields parametrizing an $\mathrm{SO}(5,5) /(\mathrm{SO}(5) \times \mathrm{SO}(5))$ coset space. For the purpose of this paper, we will only consider the linearized (free) theory with no couplings among the different types of matter.

The linearized spin-2 sector carries the symmetric Pauli-Fierz field $h_{\hat{\mu} \hat{\nu}}$. With the linearized Riemann tensor given by

$$
R_{\hat{\mu} \hat{\nu}, \hat{\rho} \hat{\sigma}}=-\partial_{\hat{\mu}} \partial_{[\hat{\rho}} h_{\hat{\sigma}] \hat{\nu}}+\partial_{\hat{\nu}} \partial_{[\hat{\rho}} h_{\hat{\sigma}] \hat{\mu}},
$$

linearization of the Einstein-Hilbert Lagrangian gives rise to the massless Fierz-Pauli Lagrangian

$$
\begin{aligned}
\mathcal{L}_{h}= & -\frac{1}{2} \partial_{\hat{\mu}} h^{\hat{\mu} \hat{\nu}} \partial_{\hat{\nu}} h_{\hat{\rho}} \hat{\rho}+\frac{1}{2} \partial_{\hat{\mu}} h^{\hat{\rho} \hat{\sigma}} \partial_{\hat{\rho}} h_{\hat{\sigma}}^{\hat{\mu}}-\frac{1}{4} \partial_{\hat{\mu}} h^{\hat{\rho} \hat{\sigma}} \partial^{\hat{\mu}} h_{\hat{\rho} \hat{\sigma}} \\
& +\frac{1}{4} \partial_{\hat{\mu}} h_{\hat{\nu}}^{\hat{\nu}} \partial^{\hat{\mu}} h_{\hat{\rho}} \hat{\rho} \\
= & -\frac{1}{4} \Omega^{\hat{\mu} \hat{\nu} \hat{\nu}} \Omega_{\hat{\mu} \hat{\nu} \hat{\rho}}+\frac{1}{2} \Omega^{\hat{\mu} \hat{\nu} \hat{\rho}} \Omega_{\hat{\nu} \hat{\rho} \hat{\mu}}+\Omega^{\hat{\mu}} \Omega_{\hat{\mu}}
\end{aligned}
$$

with $\Omega_{\hat{\mu} \hat{\nu} \hat{\rho}} \equiv \partial_{[\hat{\mu}} h_{\hat{\nu}] \hat{\rho}}$. The vector fields $A_{\hat{\mu}}{ }^{i}$ couple with a standard Maxwell term

$$
\mathcal{L}_{A}=-\frac{1}{4} F_{\hat{\mu} \hat{\nu}}^{i} F^{\hat{\mu} \hat{\nu} i}, \quad i=1, \ldots, 16,
$$

for $F_{\hat{\mu} \hat{\nu}}{ }^{i}=2 \partial_{[\hat{\mu}} A_{\hat{\nu}]}{ }^{i}$, while scalar couplings take the form

$$
\mathcal{L}_{\phi}=-\frac{1}{2} \partial_{\hat{\mu}} \phi^{\alpha} \partial^{\hat{\mu}} \phi^{\alpha}, \quad \alpha=1, \ldots, 25 .
$$

The couplings (2.6) and (2.7) break the global $\mathrm{SO}(5,5)$ symmetry of the nonlinear theory down to its compact part
$\mathrm{SO}(5) \times \mathrm{SO}(5)$, as expected for the free theory. Finally, the two-forms $B_{\hat{\mu} \hat{\nu}}{ }^{p}$ couple with a standard kinetic term

$$
\mathcal{L}_{B}=-\frac{1}{6} H_{\hat{\mu} \hat{\nu} \hat{\rho}}^{q} H^{\hat{\mu} \hat{\nu} \hat{\rho} q}, \quad q=1, \ldots, 5,
$$

for $H_{\hat{\mu} \hat{\nu} \hat{\rho}}^{q}=3 \partial_{[\hat{\mu}} B_{\hat{\nu} \hat{\rho}]}^{q}$. For the following it will be convenient to combine these fields together with their magnetic duals into a set of 10 two-forms $B_{\hat{\mu} \hat{\nu}}{ }^{a}$, satisfying first-order (anti-)self-duality field equations

$\delta_{a b} H_{\hat{\mu} \hat{\nu} \hat{\rho}}^{b}=\frac{1}{6} \varepsilon_{\hat{\mu} \hat{\nu} \hat{\rho} \hat{\sigma} \hat{\kappa} \hat{\lambda}} \eta_{a b} H^{\hat{\sigma} \hat{\kappa} \hat{\lambda} b}, \quad a=1, \ldots, 10$,

with the $\mathrm{SO}(5,5)$ invariant constant tensor $\eta_{a b}$. Equations (2.9) amount to a description of these degrees of freedom in terms of 5 self-dual and 5 anti-self-dual two-forms.

\section{B. The $\mathcal{N}=(3,1)$ model}

Let us now turn to the free field equations associated with the $\mathcal{N}=(3,1)$ multiplet $(2.2 b)$. This multiplet does not carry a standard graviton field, but an exotic three-index tensor field of mixed-symmetry type [19]

$$
\text { : } C_{\hat{\mu} \hat{\nu}, \hat{\rho}}=-C_{\hat{\nu} \hat{\mu}, \hat{\rho}}, \quad C_{[\hat{\mu} \hat{\nu}, \hat{\rho}]}=0 .
$$

Its field equation is given by a self-duality equation [1]

$$
S_{\hat{\mu} \hat{\nu} \hat{\rho}, \hat{\sigma} \hat{\tau}}=\frac{1}{6} \varepsilon_{\hat{\mu} \hat{\nu} \hat{\rho} \hat{\eta} \hat{\kappa} \hat{\lambda}} S^{\hat{\eta} \hat{\kappa} \hat{\lambda}} \hat{\sigma} \hat{\tau},
$$

in terms of its second-order curvature

$$
S_{\hat{\mu} \hat{\nu} \hat{\rho}, \hat{\sigma} \hat{\imath}}=3 \partial_{\hat{\sigma}} \partial_{[\hat{\mu}} C_{\hat{\nu} \hat{\rho}], \hat{\imath}}-3 \partial_{\hat{\tau}} \partial_{[\hat{\mu}} C_{\hat{\nu} \hat{\rho}], \hat{\sigma}} .
$$

Counting reveals that the field equation (2.11) captures the 8 degrees of freedom as counted in the multiplet (2.2b). Moreover, curvature and field equation are invariant under the gauge symmetries

$$
\delta C_{\hat{\mu} \hat{\nu}, \hat{\rho}}=2 \partial_{[\hat{\mu}} \alpha_{\hat{\nu}] \hat{\rho}}+\partial_{\hat{\rho}} \beta_{\hat{\mu} \hat{\nu}}-\partial_{[\hat{\rho}} \beta_{\hat{\mu} \hat{\nu}]}
$$

with parameters $\alpha_{\hat{\mu} \hat{\nu}}=\alpha_{(\hat{\mu} \hat{\nu})}$ and $\beta_{\hat{\mu} \hat{\nu}}=\beta_{[\hat{\mu} \hat{\nu}]}$. An action principle for the field equations (2.11) has been constructed in [16] based on the prepotential formalism introduced in [17] in the context of linearized gravity.

In addition to the exotic tensor field, the bosonic field content of the $\mathcal{N}=(3,1)$ multiplet $(2.2 b)$ contains 14 vectors, 12 self-dual two-forms and 28 scalar fields. The dynamics of vector and scalar fields can be captured by standard Lagrangians (2.6) and (2.7) (with different range of internal indices). The self-dual two-forms $B_{\hat{\mu} \hat{\nu}}{ }^{a}$ obey a self-duality equation similar to (2.9) 


$$
H_{\hat{\mu} \hat{\nu} \hat{\rho}}^{a}=\frac{1}{6} \varepsilon_{\hat{\mu} \hat{\nu} \hat{\rho} \hat{\sigma} \hat{\kappa} \hat{\lambda}} H^{\hat{\sigma} \hat{\kappa} \hat{\lambda} a}, \quad a=1, \ldots, 12,
$$

contrary to (2.9), no indefinite tensor $\eta_{a b}$ appears in this equation, and all forms are self-dual. ${ }^{1}$ As a consequence there is no standard action principle for these field equations; they can however be derived from an action with nonmanifest Lorentz invariance [13] or upon coupling to an auxiliary scalar field [20]. ${ }^{2}$

The free $\mathcal{N}=(3,1)$ theory is invariant under the $R$-symmetry group $\mathrm{USp}(6) \times \mathrm{USp}(2)$. The (yet elusive) interacting theory is conjectured to exhibit a global $\mathrm{F}_{4(4)}$ symmetry with in particular the 28 scalars parametrizing the coset space $\mathrm{F}_{4(4)} /(\mathrm{USp}(6) \times \mathrm{USp}(2))$ [1]

\section{The $\mathcal{N}=(4,0)$ model}

The $\mathcal{N}=(4,0)$ multiplet carries an exotic four-index tensor field with the symmetries of the Riemann tensor

$$
: \quad T_{\hat{\mu} \hat{\nu}, \hat{\rho} \hat{\sigma}}=T_{\hat{\rho} \hat{\sigma}, \hat{\mu} \hat{\nu}}=-T_{\hat{\nu} \hat{\mu}, \hat{\rho} \hat{\sigma}}, \quad T_{[\hat{\mu} \hat{\nu}, \hat{\rho}] \hat{\sigma}}=0 .
$$

Its field equation is given by a self-duality equation [1]

$$
G_{\hat{\mu} \hat{\nu} \hat{\lambda}, \hat{\rho} \hat{\sigma} \hat{\tau}}=\frac{1}{6} \varepsilon_{\hat{\mu} \hat{\nu} \hat{\nu} \hat{\alpha} \hat{\beta} \hat{\gamma}} G^{\hat{\alpha} \hat{\beta} \hat{\gamma}} \hat{\rho} \hat{\sigma} \hat{\tau},
$$

in terms of its second-order curvature

$$
G_{\hat{\mu} \hat{\nu} \hat{\lambda}, \hat{\rho} \hat{\sigma} \hat{\imath}}=3 \partial_{\hat{\rho}} \partial_{[\hat{\mu}} T_{\hat{\nu} \hat{\lambda}], \hat{\sigma} \hat{\imath}}+3 \partial_{\hat{\sigma}} \partial_{[\hat{\mu}} T_{\hat{\nu} \hat{\lambda}], \hat{\imath} \hat{\rho}}+3 \partial_{\hat{\tau}} \partial_{[\hat{\mu}} T_{\hat{\nu} \hat{\lambda}], \hat{\rho} \hat{\sigma}} .
$$

Counting confirms that this field equation describes the 5 degrees of freedom as counted in the multiplet (2.2c). Moreover, curvature and field equation are invariant under the gauge symmetries

$$
\delta T_{\hat{\mu} \hat{\nu}, \hat{\rho} \hat{\sigma}}=\partial_{[\hat{\mu}} \lambda_{\hat{\nu}], \hat{\rho} \hat{\sigma}}+\partial_{[\hat{\rho}} \lambda_{\hat{\sigma}], \hat{\mu} \hat{\nu}},
$$

with the $(2,1)$ gauge parameter $\lambda_{\hat{\mu}, \hat{\rho} \hat{\sigma}}=\lambda_{\hat{\mu},[\hat{\rho} \hat{\sigma}]}, \lambda_{[\hat{\mu}, \hat{\rho} \hat{\sigma}]}=0$. An action principle for (2.16) has been constructed in $[14,15]$ based on the prepotential formalism of [17]. The bosonic part of the $\mathcal{N}=(4,0)$ multiplet (2.2c) combines the exotic tensor field $T_{\hat{\mu} \hat{\nu}, \hat{\rho} \hat{\sigma}}$ with 42 scalars and 27 self-dual two-forms. Their dynamics is described by a free Lagrangian (2.7) and self-duality equations (2.14), respectively.

\footnotetext{
${ }^{1}$ For uniformity, we use the same indices $a, b$, to label twoforms in all three models, despite the fact that the range of these indices differs among the different models according to the number of two-form fields. This should not be a source of confusion.

${ }^{2}$ For more recent constructions, see also [21,22].
}

The free $\mathcal{N}=(4,0)$ theory is invariant under the $R$-symmetry group $\mathrm{USp}(8)$. The (yet elusive) interacting theory is conjectured to exhibit a global $\mathrm{E}_{6(6)}$ symmetry with in particular the 42 scalars parametrizing the coset space $\mathrm{E}_{6(6)} / \mathrm{USp}(8)[1]$.

\section{5 + 1 SPLIT}

Upon dimensional reduction to $D=5$ dimensions, the three models discussed in the previous section all reduce to the same theory: the free limit of maximal $D=5$ supergravity $[1,2]$. The bosonic sector of this theory carries a spin-2 field and 27 vector fields together with 42 scalar fields. In particular, the exotic tensor fields of the $\mathcal{N}=$ $(3,1)$ and the $\mathcal{N}=(4,0)$ model after dimensional reduction carry the $D=5$ dual graviton and double dual graviton, respectively. Within the free theory, these fields can be dualized into the standard Pauli-Fierz field $[1,23,24]$, and do not represent independent degrees of freedom. In order to make the equivalence explicit, the fields of $D=5$ supergravity (together with their on-shell duals) have to be properly identified among the various components of the $D=6$ fields.

In this section, we discuss for every of the three models the reorganization of the $D=6$ fields which allows their identification after reduction to five dimensions. However, throughout this section (and this paper) we keep the full dependence of all fields on six space-time coordinates. More precisely, we break six-dimensional Poincaré invariance down to $5+1$ and perform a standard Kaluza-Klein decomposition on the six-dimensional fields without dropping the dependence on the sixth coordinate. We then rearrange the equations such that they take the form of the five-dimensional (free) supergravity equations however sourced by derivatives of matter fields along the sixth direction. The resulting reformulation of the sixdimensional models casts their dynamics into a common framework-which ultimately allows us to construct uniform actions for the three models.

For the purpose of this paper, we choose the $5+1$ coordinate split

$$
\left\{x^{\hat{\mu}}\right\} \rightarrow\left\{x^{\mu}, y\right\}, \quad \mu=0, \ldots, 4,
$$

by singling out one of the spatial coordinates. Of course, an analogous construction can be performed with a split along the timelike coordinate which may be of interest for example in a Hamiltonian context.

\section{A. The $\mathcal{N}=(2,2)$ model}

With the coordinate split (3.1), we parametrize the graviton of the $\mathcal{N}=(2,2)$ theory as

$$
h_{\hat{\mu} \hat{\nu}}=\left(\begin{array}{cc}
h_{\mu \nu}-\frac{1}{3} \eta_{\mu \nu} \phi & A_{\mu} \\
A_{\nu} & \phi
\end{array}\right) \text {, }
$$


which is the linearized form of the standard Kaluza-Klein reduction ansatz. Recall that all fields still depend on six coordinates. Working out the Lagrangian (2.5) in this parametrization gives rise to its expression

$$
\begin{aligned}
\mathcal{L}_{h} \longrightarrow \mathcal{L}_{\square}= & -\frac{1}{2} \partial_{\mu} h^{\mu \nu} \partial_{\nu} h_{\rho}{ }^{\rho}+\frac{1}{2} \partial_{\mu} h^{\rho \sigma} \partial_{\rho} h_{\sigma}{ }^{\mu}-\frac{1}{4} \partial_{\mu} h^{\nu \rho} \partial^{\mu} h_{\nu \rho}+\frac{1}{4} \partial_{\mu} h_{\nu}{ }^{\nu} \partial^{\mu} h_{\rho}{ }^{\rho} \\
& -\frac{1}{4} \partial_{y} h^{\mu \nu} \partial_{y} h_{\mu \nu}+\partial_{y} h^{\mu \nu} \partial_{\mu} A_{\nu}-\partial_{y} h_{\sigma}{ }^{\sigma} \partial^{\rho} A_{\rho}+\frac{1}{4} \partial_{y} h_{\sigma}{ }^{\sigma} \partial_{y} h_{\rho}{ }^{\rho}-\frac{2}{3} \partial_{y} h_{\sigma}{ }^{\sigma} \partial_{y} \phi \\
& -\frac{1}{4} F^{\mu \nu} F_{\mu \nu}-\frac{1}{3} \partial^{\mu} \phi \partial_{\mu} \phi+\frac{4}{3} \partial_{y} A^{\mu} \partial_{\mu} \phi+\frac{5}{9} \partial_{y} \phi \partial_{y} \phi,
\end{aligned}
$$

up to total derivatives. As an illustration of the above discussion let us note the explicit form of the equations for the fivedimensional spin-2 field

$$
\mathcal{G}_{\mu \nu}=-\frac{1}{2} \partial_{y} \partial_{y} h_{\mu \nu}+\frac{1}{2} \eta_{\mu \nu} \partial_{y} \partial_{y} h_{\rho}^{\rho}-\frac{2}{3} \eta_{\mu \nu} \partial_{y} \partial_{y} \phi
$$

in terms of the linearized Einstein tensor

$$
\begin{aligned}
\mathcal{G}_{\mu \nu}= & -\partial^{\rho} D_{(\mu} h_{\nu) \rho}+\frac{1}{2} \partial_{\rho} D^{\rho} h_{\mu \nu}+\frac{1}{2} \partial_{(\mu} D_{\nu)} h_{\rho}{ }^{\rho}+\frac{1}{2} \eta_{\mu \nu} \partial^{\rho} D^{\sigma} h_{\rho \sigma}-\frac{1}{2} \eta_{\mu \nu} \partial_{\rho} D^{\rho} h_{\sigma}{ }^{\sigma}, \\
& \text { with covariant derivatives } D_{\mu} h_{\nu \rho} \equiv \partial_{\mu} h_{\nu \rho}-\frac{2}{3} \partial_{y} A_{\mu} \eta_{\nu \rho} .
\end{aligned}
$$

The form of (3.4) shows that upon dimensional reduction to $D=5$ dimensions, these equations reproduce the (linearized) five-dimensional Einstein field equations. In contrast, the coordinate dependence along the sixth coordinate induces a nontrivial gauge structure via covariant derivatives (3.5) and nonvanishing source terms in (3.4). This is very much in the spirit of the reformulation of higher-dimensional supergravities as exceptional field theories. Indeed, Eq. (3.4) can be equivalently obtained upon linearizing the corresponding $\mathrm{E}_{6(6)}$ ExFT $[10,11]$ upon proper identification of the coordinate $y$ among the 27 internal coordinates on which this ExFT is based.

Let us also note that the Lagrangian (3.3) can be put to the more compact form

$$
\begin{aligned}
\mathcal{L}_{\square}= & -\frac{1}{4} \Omega^{\mu \nu \rho} \Omega_{\mu \nu \rho}+\frac{1}{2} \Omega^{\mu \nu \rho} \Omega_{\nu \rho \mu}+\Omega^{\mu} \Omega_{\mu}-\frac{1}{3}\left(\partial^{\mu} \phi-2 \partial_{y} A^{\mu}\right)\left(\partial_{\mu} \phi-2 \partial_{y} A_{\mu}\right) \\
& -\frac{1}{4} F^{\mu \nu} F_{\mu \nu}+\frac{5}{9} \partial_{y} \phi \partial_{y} \phi-\frac{2}{3} \partial_{y} h_{\sigma}{ }^{\sigma} \partial_{y} \phi+\frac{1}{4} \partial_{y} h_{\sigma}{ }^{\sigma} \partial_{y} h_{\rho}{ }^{\rho}-\frac{1}{4} \partial_{y} h^{\mu \nu} \partial_{y} h_{\mu \nu},
\end{aligned}
$$

with the linearized (and covariantized) anholonomity objects

$$
\Omega_{\mu \nu \rho} \equiv \partial_{[\mu} h_{\nu] \rho}-\frac{2}{3} \partial_{y} A_{[\mu} \eta_{\nu] \rho}, \quad \Omega_{\mu} \equiv \Omega_{\mu \nu}{ }^{\nu}
$$

The remaining part of the six-dimensional degrees of freedom described by (3.6) are captured by a (modified) five-dimensional Maxwell and Klein-Gordon equation for $A_{\mu}$ and $\phi$, respectively, obtained by varying (3.6). It is useful to note the symmetries of the Lagrangian (3.6) descending from six-dimensional spin-2 gauge transformations

$$
\begin{aligned}
\delta h_{\mu \nu} & =2 \partial_{(\mu} \xi_{\nu)}+\frac{2}{3} \eta_{\mu \nu} \partial_{y} \lambda, \\
\delta A_{\mu} & =\partial_{\mu} \lambda+\partial_{y} \xi_{\mu}, \\
\delta \phi & =2 \partial_{y} \lambda,
\end{aligned}
$$

upon decomposition of the six-dimensional gauge parameter as $\left\{\xi^{\hat{\mu}}\right\}=\left\{\xi^{\mu}, \lambda\right\}$.

In a similar way, the six-dimensional Maxwell and Klein-Gordon Lagrangians (2.6) and (2.7) take the form

$$
\begin{aligned}
& \mathcal{L}_{A}=-\frac{1}{4} F^{\mu \nu i} F_{\mu \nu}{ }^{i}-\frac{1}{2}\left(\partial^{\mu} \phi^{i}-\partial_{y} A^{\mu i}\right)\left(\partial_{\mu} \phi^{i}-\partial_{y} A_{\mu}{ }^{i}\right), \\
& \mathcal{L}_{\phi}=-\frac{1}{2} \partial^{\mu} \phi^{\alpha} \partial_{\mu} \phi^{\alpha}-\frac{1}{2} \partial_{y} \phi^{\alpha} \partial_{y} \phi^{\alpha}
\end{aligned}
$$


respectively, after splitting $\left\{A_{\hat{\mu}}{ }^{i}\right\}=\left\{A_{\mu}{ }^{i}, \phi^{i}\right\}$, and with Abelian $F_{\mu \nu}{ }^{i}=2 \partial_{[\mu} A_{\nu]}{ }^{i}$, giving rise to modified Maxwell and Klein-Gordon equations for their components. The rewriting of the tensor field sector is slightly less straightforward: rather than evaluating the Lagrangian (2.8), we choose to evaluate the first-order field equations (2.9) after splitting the 6D tensor fields into $\left\{B_{\hat{\mu} \hat{\nu}}{ }^{a}\right\}=\left\{B_{\mu \nu}{ }^{a}, B_{\mu 5}{ }^{a} \equiv A_{\mu}{ }^{a}\right\}$

$$
\eta_{a b} H_{\mu \nu \rho}^{b}=\frac{1}{2} \varepsilon_{\mu \nu \rho \kappa \lambda} \delta_{a b}\left(F^{\kappa \lambda b}+\partial_{y} B^{\kappa \lambda b}\right)
$$

where we use conventions $\varepsilon_{\mu \nu \rho \kappa \lambda \zeta}=\varepsilon_{\mu \nu \rho \kappa \lambda}$, and Abelian field strengths $H_{\mu \nu \rho}{ }^{a}=3 \partial_{[\mu} B_{\nu \rho]}{ }^{a}$, and $F_{\mu \nu}{ }^{a}=2 \partial_{[\mu} A_{\nu]}{ }^{a}$, respectively. These equations can be integrated to a Lagrangian

$$
\mathcal{L}_{日}=-\frac{1}{4}\left(F_{\mu \nu}^{a}+\partial_{y} B_{\mu \nu}{ }^{a}\right)\left(F^{\mu \nu a}+\partial_{y} B^{\mu \nu a}\right)-\frac{1}{24} \varepsilon^{\mu \nu \rho \sigma \tau} \eta_{a b} \partial_{y} B_{\mu \nu}{ }^{a} H_{\rho \sigma \tau}{ }^{b} .
$$

Again, this Lagrangian can be deduced from the linearized version of exceptional field theory. We discuss this mechanism in more detail in Appendix A 2. As we will see in the following, this form of the Lagrangian allows for the most uniform treatment of the different six-dimensional models. After dimensional reduction to $D=5$ dimensions, it simply reduces to a collection of Maxwell terms, such that all degrees of freedom of (2.8) are described as massless vector fields in five dimensions. In the presence of the sixth dimension, the Lagrangian (3.11) gives rise to modified Maxwell equations while variation with respect to the tensor fields $B_{\mu \nu}{ }^{a}$ induces Eqs. (3.10) (under $\partial_{y}$ derivative) as duality equations relating vector and tensor fields.

In summary, the $D=6 \mathcal{N}=(2,2)$, model can be equivalently reformulated in terms of a Lagrangian given by the sum of (3.6), (3.9), and (3.11). Upon dimensional reduction to five dimensions, i.e., setting $\partial_{y} \rightarrow 0$, and rescaling of the scalar fields, this Lagrangian reduces to

$$
\begin{aligned}
\mathcal{L}_{5 D}= & -\frac{1}{4} \stackrel{\circ}{\Omega}^{\mu \nu \rho} \stackrel{\circ}{\Omega}_{\mu \nu \rho}+\frac{1}{2} \stackrel{\rho}{\Omega}^{\mu \nu \rho} \stackrel{\circ}{\Omega}_{\nu \rho \mu}+\stackrel{\circ}{\Omega}^{\mu} \stackrel{\circ}{\Omega}_{\mu} \\
& -\frac{1}{2} \partial^{\mu} \phi^{A} \partial_{\mu} \phi^{A}-\frac{1}{4} F^{\mu \nu M} F_{\mu \nu}{ }^{M}, \\
M= & 1, \ldots, 27, \quad A=1, \ldots, 42,
\end{aligned}
$$

with $\stackrel{\circ}{\Omega}_{\mu \nu \rho} \equiv \partial_{[\mu} h_{\nu] \rho}$, and where we have combined the various vector and scalar fields into joint objects

$$
\left\{A_{\mu}{ }^{M}\right\}, M=1, \ldots, 27, \quad\left\{\phi^{A}\right\}, A=1, \ldots, 42 .
$$

The Lagrangian (3.12) is the free limit of $D=5$ maximal supergravity [25]. In the interacting theory, the fields (3.13) transform in the fundamental and a nonlinear representation of its global symmetry group $\mathrm{E}_{6(6)}$.

\section{B. The $\mathcal{N}=(3,1)$ model}

We now turn to the $\mathcal{N}=(3,1)$ model. Its most characteristic element is the mixed-symmetry tensor field $C_{\hat{\mu} \hat{\nu}, \hat{\rho}}$ whose field equation (2.11) cannot be derived from a standard action principle. We thus perform the KaluzaKlein reorganization of the model on the level of the field equations. To this end, we again split coordinates as (3.1) and parametrize the mixed-symmetry tensor as

$\left\{C_{\hat{\mu} \hat{\nu}, \hat{\rho}}\right\}=\left\{C_{\mu \nu, \rho}-2 A_{[\mu} \eta_{\nu] \rho} ; C_{\mu 5, \nu}=h_{\mu \nu}+B_{\mu \nu} ; C_{\mu 5,5}=2 A_{\mu}\right\}$,

with symmetric $h_{\mu \nu}=h_{\nu \mu}$, antisymmetric $B_{\mu \nu}=-B_{\nu \mu}$, and a $(2,1)$ tensor $C_{\mu \nu, \rho}$. After dimensional reduction to five dimensions, the fields $h_{\mu \nu}$ and $A_{\mu}$ satisfy the linearized Einstein and Maxwell equations while the fields $C_{\mu \nu, \rho}$ and $B_{\mu \nu}$ describe their on-shell duals, together accounting for the 8 degrees of freedom of the six-dimensional tensor field. Explicitly, in the parametrization (3.14), the sixdimensional self-duality equations (2.11) split into two equations

$$
\begin{aligned}
& \partial_{\rho}\left(F_{\mu \nu}+\frac{1}{6} \varepsilon_{\mu \nu \lambda \sigma \tau} H^{\lambda \sigma \tau}\right) \\
& =\partial_{y} \partial_{[\mu} h_{\nu] \rho}+\frac{1}{4} \varepsilon_{\mu \nu \kappa \lambda \tau} \partial_{y} \partial^{\kappa} C_{\rho}^{\lambda \tau}+\frac{1}{2} \partial_{y} \partial_{y} C_{\mu \nu, \rho}-\partial_{y} \partial_{y} A_{[\mu} \eta_{\nu] \rho} \\
& \quad-\frac{1}{4} \varepsilon_{\rho \mu \nu \sigma \tau} \partial_{y} F^{\sigma \tau}+\partial_{y} \partial_{[\mu} B_{\nu] \rho}-\partial_{y} \partial_{\rho} B_{\mu \nu}
\end{aligned}
$$

and

$$
\begin{aligned}
R_{\mu \nu, \rho \sigma}= & \frac{1}{2} \partial_{\rho}\left(H_{\mu \nu \sigma}-\frac{1}{2} \varepsilon_{\mu \nu \sigma \kappa \lambda} F^{\kappa \lambda}\right) \\
& -\frac{1}{2} \partial_{\sigma}\left(H_{\mu \nu \rho}-\frac{1}{2} \varepsilon_{\mu \nu \rho \kappa \lambda} F^{\kappa \lambda}\right)+\frac{1}{2} \varepsilon_{\mu \nu \kappa \lambda \tau} \partial^{\kappa} \partial_{[\rho} C_{\sigma]}^{\lambda \tau} \\
& +\frac{1}{2} \partial_{y} \partial_{\rho} C_{\mu \nu, \sigma}-\frac{1}{2} \partial_{y} \partial_{\sigma} C_{\mu \nu, \rho}-\partial_{y} \partial_{\rho} A_{[\mu} \eta_{\nu] \sigma} \\
& +\partial_{y} \partial_{\sigma} A_{[\mu} \eta_{\nu] \rho},
\end{aligned}
$$

with Abelian field strengths $F_{\mu \nu}=2 \partial_{[\mu} A_{\nu]}, H_{\mu \nu \rho}=3 \partial_{[\mu} B_{\nu \rho]}$, and the linearized Riemann tensor $R_{\mu \nu, \rho \sigma}$ defined as in (2.4) however for the field $h_{\mu \nu}$. Contraction of (3.16) gives rise to an equation 
$\mathcal{G}_{\mu \nu}=-\frac{1}{2} \partial_{y} \partial^{\rho} C_{\rho(\mu, \nu)}-\frac{1}{2} \partial_{y} \partial_{(\mu} C_{\nu) \rho}{ }^{\rho}+\frac{1}{2} \eta_{\mu \nu} \partial_{y} \partial^{\rho} C_{\rho \sigma}{ }^{\sigma}$,

where $\mathcal{G}_{\mu \nu}$ denotes the linearized Einstein tensor defined as in (3.5), however with covariant derivatives now given by

$$
D_{\mu} h_{\nu \rho} \equiv \partial_{\mu} h_{\nu \rho}-\partial_{y} A_{\mu} \eta_{\nu \rho}
$$

i.e., with a different value of the coupling constant (which could be absorbed into rescaling the vector field). Equation (3.17) confirms that upon reduction to five dimensions $\left(\partial_{y} \rightarrow 0\right)$, the field $h_{\mu \nu}$ satisfies the linearized Einstein equations. As in the $\mathcal{N}=(2,2)$ model, the coordinate dependence along the sixth coordinate induces a nontrivial gauge structure (3.18) together with nonvanishing source terms in (3.17) - which differ from those of (3.4) illustrating the inequivalence of the $\mathcal{N}=(2,2)$ and the $\mathcal{N}=(3,1)$ model before dimensional reduction.

The full field equation (3.16) takes the form of a vanishing curl (in $[\rho \sigma]$ ) and can locally be integrated into the first-order equation ${ }^{3}$

$$
\begin{aligned}
\partial_{[\mu} h_{\nu] \rho}+\frac{1}{4} \varepsilon_{\mu \nu \kappa \lambda \tau} \partial^{\kappa} C_{\rho}^{\lambda \tau}+\frac{1}{2}\left(H_{\mu \nu \rho}-\frac{1}{2} \varepsilon_{\mu \nu \rho \kappa \lambda} F^{\kappa \lambda}\right) \\
+\frac{1}{2} \partial_{y} C_{\mu \nu, \rho}-\partial_{y} A_{[\mu} \eta_{\nu] \rho}=\partial_{\rho} u_{\mu \nu}
\end{aligned}
$$

with an antisymmetric tensor $u_{\mu \nu}=-u_{\nu \mu}$. Combining this equation with the field equation (3.15) implies that

$\partial_{\rho}\left(F_{\mu \nu}+\frac{1}{6} \varepsilon_{\mu \nu \kappa \lambda \tau} H^{\kappa \lambda \tau}+\frac{3}{2} \partial_{y} B_{\mu \nu}-\partial_{y} u_{\mu \nu}\right)=0$,

which can be further integrated into another first-order duality equation

$$
F_{\mu \nu}+\frac{1}{6} \varepsilon_{\mu \nu \kappa \lambda \tau} H^{\kappa \lambda \tau}+\frac{3}{2} \partial_{y} B_{\mu \nu}-\partial_{y} u_{\mu \nu}=0,
$$

up to a function $f_{\mu \nu}(y)$ that can be absorbed into $u_{\mu \nu}$. Eventually, we can use (3.21) to bring (3.19) into the form

$$
\begin{aligned}
& \partial_{[\mu} h_{\nu] \rho}+\frac{1}{4} \varepsilon_{\mu \nu \kappa \lambda \tau} \partial^{\kappa} C_{\rho}^{\lambda \tau}-\partial_{\rho} u_{\mu \nu} \\
& =\frac{1}{4} \varepsilon_{\mu \nu \rho \kappa \lambda} \partial_{y}\left(u^{\kappa \lambda}-\frac{3}{2} B^{\kappa \lambda}\right)-\frac{1}{2} \partial_{y} C_{\mu \nu, \rho}+\partial_{y} A_{[\mu} \eta_{\nu] \rho} .
\end{aligned}
$$

To sum up, we have cast the original second-order field equations (2.11) of the six-dimensional mixed-symmetry

\footnotetext{
${ }^{3}$ Here, and in the following we work locally and ignore potential subtleties that may arise from a nontrivial topology. We refer to [26] for a discussion of such issues in the context of chiral $p$-forms.
}

tensor field into the form of two first-order duality equations (3.21) and (3.22), upon parametrizing the sixdimensional fields in terms of its components (3.14) and introduction of an additional field $u_{\mu \nu}$. Upon reduction to five dimensions, these equations constitute the duality equations relating the vector-tensor fields, and the graviton-dual graviton fields, respectively.

It is instructive to work out the gauge symmetries of these equations which originate from the $D=6$ gauge transformations (2.13). Parametrizing the six-dimensional gauge parameters as

$$
\begin{aligned}
& \alpha_{\hat{\mu} \hat{\nu}}=\left(\begin{array}{cc}
\alpha_{\mu \nu}-\eta_{\mu \nu} \lambda & \frac{1}{2}\left(\xi_{\mu}+3 \Lambda_{\mu}\right) \\
\frac{1}{2}\left(\xi_{\mu}+3 \Lambda_{\mu}\right) & 2 \lambda
\end{array}\right), \\
& \beta_{\hat{\mu} \hat{\nu}}=\left(\begin{array}{cc}
\beta_{\mu \nu} & \frac{3}{2}\left(\xi_{\mu}-\Lambda_{\mu}\right) \\
\frac{3}{2}\left(\Lambda_{\mu}-\xi_{\mu}\right) & 0
\end{array}\right),
\end{aligned}
$$

their action on the various components of (3.14) is derived as

$$
\begin{aligned}
\delta A_{\mu} & =\partial_{\mu} \lambda+\frac{1}{2} \partial_{y}\left(\xi_{\mu}-3 \Lambda_{\mu}\right), \\
\delta B_{\mu \nu} & =2 \partial_{[\mu} \Lambda_{\nu]}+\frac{1}{3} \partial_{y} \beta_{\mu \nu}, \\
\delta h_{\mu \nu} & =2 \partial_{(\mu} \xi_{\nu)}+\eta_{\mu \nu} \partial_{y} \lambda-\partial_{y} \alpha_{\mu \nu}, \\
\delta C_{\mu \nu, \rho} & =2 \partial_{[\mu} \alpha_{\nu] \rho}+\partial_{\rho} \beta_{\mu \nu}-\partial_{[\rho} \beta_{\mu \nu]}+\partial_{y}\left(\xi_{[\mu} \eta_{\nu] \rho}-3 \Lambda_{[\mu} \eta_{\nu] \rho}\right) .
\end{aligned}
$$

With the field $u_{\mu \nu}$ defined by Eq. (3.19), its gauge variation is found by integrating up the variation of (3.19) and takes the form

$$
\delta u_{\mu \nu}=\partial_{[\mu} \xi_{\nu]}+\frac{1}{6} \varepsilon_{\mu \nu \rho \sigma \tau} \partial^{\rho} \beta^{\sigma \tau}+\frac{1}{2} \partial_{y} \beta_{\mu \nu}
$$

For later use, let us note that contraction of (3.22) with the fully antisymmetric $\varepsilon$-tensor yields

$$
\frac{1}{6} \partial^{\rho} C_{\mu \nu, \rho}+\frac{1}{3} \partial_{[\mu} C_{\nu] \rho}{ }^{\rho}+\frac{1}{6} \varepsilon_{\mu \nu \rho \sigma \tau} \partial^{\rho} u^{\sigma \tau}=\frac{1}{2} \partial_{y}\left(u_{\mu \nu}-\frac{3}{2} B_{\mu \nu}\right),
$$

while contraction gives rise to

$$
\partial_{\mu} h_{\nu}{ }^{\mu}-\partial_{\nu} h_{\mu}{ }^{\mu}+\partial_{y} C_{\mu \nu}{ }^{\mu}+4 \partial_{y} A_{\nu}=2 \partial^{\mu} u_{\mu \nu} .
$$

This gives rise to an equivalent rewriting of (3.22) as 


$$
\begin{aligned}
\partial_{[\mu} h_{\nu] \rho}+\partial_{\sigma} h_{[\mu}{ }^{\sigma} \eta_{\nu] \rho}+\eta_{\rho[\mu} \partial_{\nu]} h_{\sigma}{ }^{\sigma} \\
=-\frac{1}{4} \varepsilon_{\mu \nu \kappa \lambda \tau} \partial^{\kappa}\left(C^{\lambda \tau} \rho+\varepsilon^{\lambda \tau \alpha \beta \sigma} u_{\alpha \beta} \eta_{\rho \sigma}\right)-3 \partial_{y} A_{[\mu} \eta_{\nu] \rho} \\
\quad+\frac{1}{4} \varepsilon_{\mu \nu \rho \kappa \lambda} \partial_{y}\left(u^{\kappa \lambda}-\frac{3}{2} B^{\kappa \lambda}\right)-\frac{1}{2} \partial_{y} C_{\mu \nu, \rho}-\partial_{y} C_{\sigma[\mu}{ }^{\sigma} \eta_{\nu] \rho} .
\end{aligned}
$$

Let us further note that taking the divergence of (3.21) yields the Maxwell type equation

$$
\begin{aligned}
\partial^{\mu} F_{\mu \nu}= & \frac{1}{2} \partial_{y} \partial_{\mu} h_{\nu}{ }^{\mu}-\frac{1}{2} \partial_{y} \partial_{\nu} h_{\mu}{ }^{\mu}+\frac{1}{2} \partial_{y} \partial_{y} C_{\mu \nu}{ }^{\mu} \\
& -\frac{3}{2} \partial_{y} \partial^{\mu} B_{\mu \nu}+2 \partial_{y} \partial_{y} A_{\nu},
\end{aligned}
$$

where we have used (3.27) in order to eliminate the divergence of $u_{\mu \nu}$.

For the remaining fields of the $\mathcal{N}=(3,1)$ model, the $5+1$ Kaluza-Klein split is achieved just as for the $\mathcal{N}=$ $(2,2)$ model discussed above. The six-dimensional field equations of the 14 vector fields and 28 scalar fields take the form obtained from variation of Lagrangians of the form (3.9), respectively. The field equations of the 12 selfdual forms take the form

$$
H_{\mu \nu \rho}^{a}=\frac{1}{2} \varepsilon_{\mu \nu \rho \kappa \lambda}\left(F^{\kappa \lambda a}+\partial_{y} B^{\kappa \lambda a}\right),
$$

after splitting the two-forms according to $\left\{B_{\hat{\mu} \hat{\nu}}{ }^{a}\right\}=$ $\left\{B_{\mu \nu}{ }^{a}, B_{\mu 5}{ }^{a} \equiv A_{\mu}{ }^{a}\right\}$. The equations may be integrated up to an action in precise analogy with (3.11), cf. the discussion in Appendix A 2.

\section{The $\mathcal{N}=(4,0)$ model}

In this model, the exotic graviton is given by the rankfour tensor (2.15) whose dynamics is defined by the selfduality equations (2.16) for its second-order curvature. According to the split of coordinates (3.1), we parametrize the various components of this field as

$$
\left\{T_{\hat{\mu} \hat{\nu}, \hat{\rho} \hat{\sigma}}\right\}=\left\{T_{\mu \nu, \rho \sigma} ; T_{\mu \nu, \rho 5}=C_{\mu \nu, \rho} ; T_{\mu 5, \nu 5}=h_{\mu \nu}\right\} .
$$

After dimensional reduction to five dimensions, these fields describe the graviton, dual graviton and double dual graviton, respectively. Explicitly, in this parametrization the six-dimensional field equations (2.16) split into two equations

$$
\begin{aligned}
R_{\mu \nu, \rho \sigma}= & \frac{1}{2} \partial_{y} \partial_{\mu} C_{\rho \sigma, \nu}-\frac{1}{2} \partial_{y} \partial_{\nu} C_{\rho \sigma, \mu}+\frac{1}{2} \partial_{y} \partial_{\rho} C_{\mu \nu, \sigma} \\
& -\frac{1}{2} \partial_{y} \partial_{\sigma} C_{\mu \nu, \rho}+\frac{1}{2} \varepsilon_{\mu \nu \kappa \lambda \tau} \partial_{[\rho} \partial^{\kappa} C_{\sigma]}^{\lambda \tau} \\
& +\frac{1}{4} \varepsilon_{\mu \nu \kappa \lambda \tau} \partial_{y} \partial^{\kappa} T_{\rho \sigma}^{\lambda \tau}+\frac{1}{2} \partial_{y} \partial_{y} T_{\mu \nu, \rho \sigma}
\end{aligned}
$$

$$
\begin{aligned}
& \varepsilon_{\mu \nu \alpha \beta \gamma} \partial^{\alpha} \partial_{[\rho} T_{\sigma \tau]}^{\beta \gamma} \\
& =-2 \partial_{\mu} \partial_{[\rho} C_{\sigma \tau], \nu}+2 \partial_{\nu} \partial_{[\rho} C_{\sigma \tau], \mu}-2 \partial_{y} \partial_{[\rho} T_{\sigma \tau], \mu \nu},
\end{aligned}
$$

with the linearized Riemann tensor $R_{\mu \nu, \rho \sigma}$ defined as in (2.4) for the field $h_{\mu \nu}$. The second equation (3.33) has the form of a curl in $[\rho \sigma \tau]$ and can be integrated up into

$\frac{1}{2} \varepsilon_{\mu \nu \alpha \beta \gamma} \partial^{\alpha} T_{\sigma \tau}^{\beta \gamma}+\partial_{\mu} C_{\sigma \tau, \nu}-\partial_{\nu} C_{\sigma \tau, \mu}+\partial_{y} T_{\sigma \tau, \mu \nu}=2 \partial_{[\sigma} v_{\tau], \mu \nu}$,

up to a tensor $v_{\tau, \mu \nu}=-v_{\tau, \nu \mu}$, determined by this equation up to the gauge freedom $\delta v_{\tau, \mu \nu}=\partial_{\tau} \zeta_{\mu \nu}$. Combining (3.34) with the first field equation (3.32), we find

$$
\begin{aligned}
R_{\mu \nu, \rho \sigma}= & \frac{1}{2} \partial_{y} \partial_{\rho} C_{\mu \nu, \sigma}-\frac{1}{2} \partial_{y} \partial_{\sigma} C_{\mu \nu, \rho}+\frac{1}{2} \varepsilon_{\mu \nu \kappa \lambda \tau} \partial_{[\rho} \partial^{\kappa} C_{\sigma]}^{\lambda \tau} \\
& +\partial_{y} \partial_{[\rho} v_{\sigma], \mu \nu},
\end{aligned}
$$

which in turn is a curl in $[\rho \sigma]$ and can be integrated up into

$\partial_{[\mu} h_{\nu] \rho}+\frac{1}{4} \varepsilon_{\mu \nu \lambda \sigma \tau} \partial^{\lambda} C_{\rho}^{\sigma \tau}+\frac{1}{2} \partial_{y} C_{\mu \nu, \rho}+\frac{1}{2} \partial_{y} v_{\rho, \mu \nu}=\partial_{\rho} u_{\mu \nu}$,

up to an antisymmetric field $u_{\mu \nu}=-u_{\nu \mu}$. As for the $\mathcal{N}=$ $(3,1)$ model, we have obtained an equivalent reformulation of the dynamics in terms of two first-order equations (3.34) and (3.36) from which the original second-order field equations (3.32), (3.33), can be obtained by derivation. After reduction to five dimensions, Eqs. (3.34) and (3.36) describe the duality relations between graviton and dual graviton and between dual graviton and double dual graviton, respectively. In particular, Eq. (3.36) differs from Eq. (3.22) in the $\mathcal{N}=(3,1)$ model only if fields depend on the sixth coordinate.

It is instructive to work out the gauge symmetries of these equations which originate from the $D=6$ gauge transformations (2.18). Parametrizing the six-dimensional gauge parameters as

$$
\left\{\lambda_{\hat{\rho}, \hat{\mu} \hat{\nu}}\right\}=\left\{\lambda_{\rho, \mu \nu} ; \lambda_{\mu, \nu 5}=2 \alpha_{\mu \nu}-\frac{2}{3} \beta_{\mu \nu} ; \lambda_{5, \mu 5}=2 \xi_{\mu}\right\},
$$

with symmetric $\alpha_{\mu \nu}$, and antisymmetric $\beta_{\mu \nu}$, their action on the various components of (3.31) is derived as

$$
\begin{aligned}
\delta h_{\mu \nu} & =2 \partial_{(\mu} \xi_{\nu)}-2 \partial_{y} \alpha_{\mu \nu} \\
\delta C_{\mu \nu, \rho} & =2 \partial_{[\mu} \alpha_{\nu] \rho}+\partial_{\rho} \beta_{\mu \nu}-\partial_{[\rho} \beta_{\mu \nu]}-\frac{1}{2} \partial_{y} \lambda_{\rho, \mu \nu}, \\
\delta T_{\mu \nu, \rho \sigma} & =\partial_{[\mu} \lambda_{\nu], \rho \sigma}+\partial_{[\rho} \lambda_{\sigma], \mu \nu} .
\end{aligned}
$$


Gauge variations of the two new fields $v_{\rho, \mu \nu}$ and $u_{\mu \nu}$ are obtained by integrating up the variation of (3.34) and (3.36), respectively, giving rise to

$$
\begin{aligned}
\delta u_{\mu \nu}= & \partial_{[\mu} \xi_{\nu]}+\frac{1}{6} \varepsilon_{\mu \nu \lambda \sigma \tau} \partial^{\lambda} \beta^{\sigma \tau}+\frac{1}{3} \partial_{y} \beta_{\mu \nu}+\frac{1}{2} \partial_{y} \zeta_{\mu \nu}, \\
\delta v_{\rho, \mu \nu}= & \frac{1}{4} \varepsilon_{\mu \nu \kappa \lambda \sigma} \partial^{\kappa} \lambda_{\rho}^{\lambda \sigma}+2 \partial_{[\mu} \alpha_{\nu] \rho}+\frac{2}{3} \partial_{[\mu} \beta_{\nu] \rho} \\
& +\partial_{\rho} \zeta_{\mu \nu}+\frac{1}{2} \partial_{y} \lambda_{\rho, \mu \nu},
\end{aligned}
$$

where the antisymmetric gauge parameter $\zeta_{\mu \nu}=-\zeta_{\nu \mu}$ has been introduced after (3.34).

Let us finally note that from (3.32) and (3.35), we may obtain the modified Einstein equations

$$
\begin{aligned}
\stackrel{\circ}{\mathcal{G}}_{\mu \nu}= & -\frac{1}{2} \partial_{y} \partial^{\rho} C_{\rho(\mu, \nu)}-\frac{1}{2} \partial_{y} \partial_{(\mu} C_{\nu) \rho}{ }^{\rho}+\frac{1}{2} \partial_{y} \partial_{\rho} v_{(\mu, \nu)}{ }^{\rho} \\
& -\frac{1}{2} \partial_{y} \partial_{(\mu} v^{\rho}{ }_{\nu) \rho}+\frac{1}{2} \eta_{\mu \nu} \partial_{y} \partial^{\rho} C_{\rho \sigma}{ }^{\sigma}-\frac{1}{2} \eta_{\mu \nu} \partial_{y} \partial_{\rho} v_{\sigma}{ }^{\sigma \rho},
\end{aligned}
$$

with the linearized Einstein tensor $\stackrel{\circ}{\mathcal{G}}_{\mu \nu}$ defined as

$$
\begin{aligned}
\stackrel{\circ}{\mathcal{G}}_{\mu \nu}= & -\partial^{\rho} \partial_{(\mu} h_{\nu) \rho}+\frac{1}{2} \partial_{\rho} \partial^{\rho} h_{\mu \nu}+\frac{1}{2} \partial_{\mu} \partial_{\nu} h_{\rho}{ }^{\rho} \\
& +\frac{1}{2} \eta_{\mu \nu} \partial^{\rho} \partial^{\sigma} h_{\rho \sigma}-\frac{1}{2} \eta_{\mu \nu} \partial_{\rho} \partial^{\rho} h_{\sigma}{ }^{\sigma},
\end{aligned}
$$

which differs from the previous models by the absence of covariant derivatives, cf. (3.5).
For the remaining fields of the $\mathcal{N}=(4,0)$ model, the $5+1$ Kaluza-Klein split is achieved just as for the previous models discussed above. The field equations of the 42 scalar fields are obtained from variation of a Lagrangian of the form $\mathcal{L}_{\phi}$ in (3.9). The field equations of the 27 self-dual forms take the form of (3.30) above, again after splitting the two-forms according to $\left\{B_{\hat{\mu} \hat{\nu}}{ }^{a}\right\}=\left\{B_{\mu \nu}{ }^{a}, B_{\mu 5}{ }^{a} \equiv A_{\mu}{ }^{a}\right\}$.

\section{ACTIONS FOR (FREE) EXOTIC GRAVITON FIELDS}

In the above, we have reformulated the dynamics of the six-dimensional exotic tensor fields in terms of firstorder differential equations upon breaking six-dimensional Poincaré invariance according to the split (3.1), and introducing some additional tensor fields. As a key property of the resulting equations, we have put the dynamics of the different models into a form which reduces to the same equations after dimensional reduction $\partial_{y} \rightarrow 0$. For example, all three models feature linearized Einstein equations for the field $h_{\mu \nu}$, given by (3.4), (3.17), and (3.40), respectively. The three equations only differ by terms carrying explicit derivatives along the sixth dimension. We will use this as a guiding principle to construct uniform Lagrangians for the $\mathcal{N}=(3,1)$ and the $\mathcal{N}=(4,0)$ model which after setting $\partial_{y} \rightarrow 0$ both reduce to the Lagrangian (3.12) of linearized $D=5$ maximal supergravity.

This construction follows the toy model of $D=6$ selfdual tensor fields whose dynamics can be described by a Lagrangian (3.11)

$$
\mathcal{L}_{\boxminus}=-\frac{1}{4}\left(F_{\mu \nu}+\partial_{y} B_{\mu \nu}\right)\left(F^{\mu \nu}+\partial_{y} B^{\mu \nu}\right)-\frac{1}{24} \varepsilon^{\mu \nu \rho \sigma \tau} \partial_{y} B_{\mu \nu} H_{\rho \sigma \tau},
$$

after a Kaluza-Klein $(5+1)$ decomposition $\left\{B_{\hat{\mu} \hat{\nu}}\right\}=$ $\left\{B_{\mu \nu}, B_{\mu 5} \equiv A_{\mu}\right\}$ of the six-dimensional tensor field. After dimensional reduction to five dimensions, the 3 degrees of freedom of the self-dual tensor field are described as a massless vector with the standard Maxwell Lagrangian to which (4.1) reduces at $\partial_{y} \rightarrow 0$. In the presence of the sixth dimension, variation of the Lagrangian (4.1) with respect to the vector field gives rise to modified Maxwell equations while variation with respect to the tensor field yields the duality equation relating $A_{\mu}$ and $B_{\mu \nu}$, which is of first order in the derivatives $\partial_{\mu}$ and appears under a global $\partial_{y}$ derivative. Combining these two equations one may infer the full six-dimensional self-duality equation. Details are spelled out in Appendix A 2. The Lagrangians for exotic gravitons are constructed in analogy to (4.1) with the role of $A_{\mu}$ and $B_{\mu \nu}$ now taken by the graviton $h_{\mu \nu}$ and its duals, respectively.

\section{A. Action for the $\mathcal{N}=(3,1)$ model}

The main result of this subsection is the following: the first-order field equations (3.21) and (3.22), which describe the dynamics of the six-dimensional exotic graviton field $C_{\hat{\mu} \hat{\nu}, \hat{\rho}}$ in the $\mathcal{N}=(3,1)$ model, can be derived from the Lagrangian

$$
\begin{aligned}
\mathcal{L}_{\boxminus}= & -\frac{1}{4} \widehat{\Omega}^{\mu \nu \rho} \widehat{\Omega}_{\mu \nu \rho}+\frac{1}{2} \widehat{\Omega}^{\mu \nu \rho} \widehat{\Omega}_{\nu \rho \mu}+\widehat{\Omega}^{\mu} \widehat{\Omega}_{\mu}-\frac{1}{16} \varepsilon^{\mu \nu \rho \sigma \tau} \partial_{y} \widehat{C}_{\mu \nu}{ }^{\lambda} \partial_{\rho} \widehat{C}_{\sigma \tau, \lambda} \\
& -\frac{3}{4} \mathcal{F}^{\mu \nu} \mathcal{F}_{\mu \nu}-\frac{9}{16} \varepsilon^{\mu \nu \rho \sigma \tau} \partial_{y} B_{\mu \nu} \partial_{\rho} B_{\sigma \tau}-\frac{3}{16} \varepsilon^{\mu \nu \rho \sigma \tau} \partial_{y} B_{\mu \nu} \partial_{y} \widehat{C}_{\rho \sigma, \tau}
\end{aligned}
$$


with

$$
\begin{aligned}
\widehat{\Omega}_{\mu \nu \rho} & \equiv \partial_{[\mu} h_{\nu] \rho}-\partial_{y} A_{[\mu} \eta_{\nu] \rho}+\frac{1}{2} \partial_{y} \widehat{C}_{\mu \nu, \rho}, \\
\widehat{C}_{\mu \nu, \rho} & \equiv C_{\mu \nu, \rho}+\varepsilon_{\mu \nu \rho \sigma \tau} u^{\sigma \tau}, \\
\mathcal{F}_{\mu \nu} & \equiv 2 \partial_{[\mu} A_{\nu]}+\frac{3}{2} \partial_{y} B_{\mu \nu} .
\end{aligned}
$$

The Lagrangian (4.2) is invariant under the gauge transformations (3.24), (3.25). After reduction to five dimensions, i.e., at $\partial_{y} \rightarrow 0$, this Lagrangian reduces to the Fierz-Pauli Lagrangian for $h_{\mu \nu}$ together with a free
Maxwell Lagrangian for $A_{\mu}$; the dual fields $\widehat{C}_{\mu \nu, \rho}$ and $B_{\mu \nu}$ drop out in this limit. In the presence of the sixth dimension, variation of the Lagrangian (4.2) with respect to the dual fields yields the first-order duality equations (3.21) and (3.22), however under an overall derivative $\partial_{y}$. Together, one recovers the full six-dimensional dynamics. Details of the equivalence are presented in Appendix B 1.

The bosonic Lagrangian for the full $\mathcal{N}=(3,1)$ model is then given by combining (4.2) with the Lagrangians of the type (3.9) and (4.1) for the remaining matter fields of the theory. Putting everything together, we obtain

$$
\begin{aligned}
\mathcal{L}_{(3,1)}= & \mathcal{L}_{\square}-\frac{1}{2}\left(\partial^{\mu} \phi^{i}-\partial_{y} A^{\mu i}\right)\left(\partial_{\mu} \phi^{i}-\partial_{y} A_{\mu}{ }^{i}\right)-\frac{1}{2} \partial^{\mu} \phi^{\alpha} \partial_{\mu} \phi^{\alpha}-\frac{1}{2} \partial_{y} \phi^{\alpha} \partial_{y} \phi^{\alpha} \\
& -\frac{1}{4} F^{\mu \nu i} F_{\mu \nu}{ }^{i}-\frac{1}{4}\left(F_{\mu \nu}{ }^{a}+\partial_{y} B_{\mu \nu}{ }^{a}\right)\left(F^{\mu \nu a}+\partial_{y} B^{\mu \nu a}\right)-\frac{1}{24} \varepsilon^{\mu \nu \rho \sigma \tau} \partial_{y} B_{\mu \nu}{ }^{a} H_{\rho \sigma \tau}{ }^{a},
\end{aligned}
$$

with indices ranging along

$$
i=1, \ldots, 14, \quad \alpha=1, \ldots, 28, \quad a=1, \ldots, 12 .
$$

After dimensional reduction to five dimensions (and rescaling of the vector field $A_{\mu}$ ), this Lagrangian coincides with the Lagrangian (3.12) of linearized maximal supergravity. The Lagrangian (4.4) describes the full six-dimensional theory, with the field content of fivedimensional maximal supergravity enhanced by the field
$\widehat{C}_{\mu \nu, \rho} . D=6$ Poincaré invariance is no longer manifest although it can still be realized on the equations of motion.

\section{B. Action for the $\mathcal{N}=(4,0)$ model}

The main result of this subsection is the following: the first-order field equations (3.34) and (3.36), which describe the dynamics of the six-dimensional exotic graviton field $T_{\hat{\mu} \hat{\nu}, \hat{\rho} \hat{\sigma}}$ in the $\mathcal{N}=(4,0)$ model, can be derived from the Lagrangian

$$
\begin{aligned}
\mathcal{L}_{\boxplus}= & -\frac{1}{4} \widehat{\Omega}^{\mu \nu \rho} \widehat{\Omega}_{\mu \nu \rho}+\frac{1}{2} \widehat{\Omega}^{\mu \nu \rho} \widehat{\Omega}_{\nu \rho \mu}+\widehat{\Omega}^{\mu} \widehat{\Omega}_{\mu}-\frac{1}{8} \varepsilon_{\mu \nu \sigma \kappa \lambda} \partial^{\mu} \widehat{C}_{\rho}^{\nu \sigma} \partial_{y} \widehat{C}^{\kappa \lambda, \rho} \\
& +\frac{1}{32} \varepsilon_{\mu \nu \sigma \kappa \lambda} \partial^{\mu} \mathscr{C}_{\rho}^{\nu \sigma} \partial_{y} \mathscr{C}^{\kappa \lambda, \rho}-\frac{1}{8} \partial_{y} \mathscr{C}_{\sigma \tau, \nu} \partial_{\mu} T^{\mu \nu, \sigma \tau}+\frac{1}{4} \partial_{y} \mathscr{C}_{\kappa \lambda, \tau} \partial^{\kappa} T^{\lambda \sigma, \tau} \sigma \\
& +\frac{1}{4} \partial_{\nu} \mathscr{C}_{\sigma \mu}{ }^{\mu} \partial_{y} T^{\sigma \tau, \nu}{ }_{\tau}-\frac{1}{8} \partial_{y} \mathscr{C}_{\sigma \mu}{ }^{\mu} \partial^{\sigma} T_{\tau \nu}{ }^{\tau \nu}-\frac{1}{64} \varepsilon_{\mu \nu \alpha \beta \gamma} \partial^{\alpha} T_{\sigma \tau}{ }^{\beta \gamma} \partial_{y} T^{\mu \nu, \sigma \tau} \\
& -\frac{1}{32} \partial_{y} T_{\sigma \tau, \mu \nu} \partial_{y} T^{\mu \nu, \sigma \tau}+\frac{1}{8} \partial_{y} T_{\sigma \mu, \nu}{ }^{\mu} \partial_{y} T_{\tau}^{\sigma \tau, \nu}-\frac{1}{32} \partial_{y} T_{\mu \nu}{ }^{\mu \nu} \partial_{y} T_{\sigma \tau}{ }^{\sigma \tau}
\end{aligned}
$$

with

$$
\begin{aligned}
\widehat{\Omega}_{\mu \nu \rho} & =\partial_{[\mu} h_{\nu] \rho}+\partial_{y} \widehat{C}_{\mu \nu, \rho}-\frac{1}{2} \partial_{y} \mathscr{C}_{\mu \nu, \rho}, \\
\widehat{C}_{\mu \nu, \rho} & =C_{\mu \nu, \rho}+\varepsilon_{\mu \nu \rho \sigma \tau} u^{\sigma \tau}, \\
\mathscr{C}_{\mu \nu, \rho} & =C_{\mu \nu, \rho}-v_{\rho, \mu \nu}+3 v_{[\rho, \mu \nu]}+2 \varepsilon_{\mu \nu \rho \sigma \tau} u^{\sigma \tau} .
\end{aligned}
$$

After reduction to five dimensions, i.e., at $\partial_{y} \rightarrow 0$, this Lagrangian reduces to the Fierz-Pauli Lagrangian for $h_{\mu \nu}$; the dual fields $\widehat{C}_{\mu \nu, \rho}, \mathscr{C}_{\mu \nu, \rho}$, and $T_{\mu \nu, \rho \sigma}$ drop out in this limit. In the presence of the sixth dimension, variation of the Lagrangian (4.6) with respect to the dual fields yields the first-order duality equations (3.34) and (3.36), however under an overall derivative $\partial_{y}$. Together, one recovers the full six-dimensional dynamics. The computation works in close analogy with the derivation for the $\mathcal{N}=(3,1)$ model, and is presented in detail in Appendix B 2.

Let us spell out the gauge transformations (3.38), (3.39) in terms of the fields (4.7) 


$$
\begin{aligned}
\delta \widehat{\Omega}_{\mu \nu \rho} & =\partial_{\rho} \partial_{[\mu} \xi_{\nu]}-\frac{2}{3} \partial_{y} \partial_{[\mu} \beta_{\nu] \rho}-\partial_{y} \partial_{[\mu} \zeta_{\nu] \rho}+\frac{1}{4} \partial_{y} \partial^{\kappa} \lambda_{[\mu}{ }^{\sigma \tau} \varepsilon_{\nu] \kappa \sigma \tau \rho}, \\
\delta \widehat{C}_{\mu \nu, \rho} & =2 \partial_{[\mu} \alpha_{\nu] \rho}-2 \partial_{[\mu} \beta_{\nu] \rho}+\varepsilon_{\mu \nu \rho \sigma \tau} \partial^{\sigma} \xi^{\tau}+\frac{1}{3} \varepsilon_{\mu \nu \rho \sigma \tau} \partial_{y}\left(\beta^{\sigma \tau}+\frac{3}{2} \zeta^{\sigma \tau}\right)-\frac{1}{2} \partial_{y} \lambda_{\rho, \mu \nu}, \\
\delta \mathscr{C}_{\mu \nu, \rho} & =2 \varepsilon_{\mu \nu \rho \sigma \tau} \partial^{\sigma} \xi^{\tau}-\frac{8}{3} \partial_{[\mu} \beta_{\nu] \rho}+2 \partial_{[\mu} \zeta_{\nu] \rho}+\frac{2}{3} \varepsilon_{\mu \nu \rho \sigma \tau} \partial_{y}\left(\beta^{\sigma \tau}+\frac{3}{2} \zeta^{\sigma \tau}\right)+\frac{1}{2} \varepsilon_{\kappa \sigma \tau \rho \mu \mu} \partial^{\kappa} \lambda_{\nu]}{ }^{\sigma \tau}-\partial_{y} \lambda_{\rho, \mu \nu},
\end{aligned}
$$

which allows us to confirm gauge invariance of the Lagrangian (4.6).

The bosonic Lagrangian for the full $\mathcal{N}=(4,0)$ model is finally given by combining (4.6) with the Lagrangians of the type (3.9) and (4.1) for the remaining matter fields of the theory. Putting everything together, we obtain

$$
\begin{aligned}
\mathcal{L}_{(4,0)}= & \mathcal{L}_{\boxplus}-\frac{1}{4}\left(F_{\mu \nu}{ }^{M}+\partial_{y} B_{\mu \nu}{ }^{M}\right)\left(F^{\mu \nu M}+\partial_{y} B^{\mu \nu M}\right)-\frac{1}{24} \varepsilon^{\mu \nu \rho \sigma \tau} \partial_{y} B_{\mu \nu}{ }^{M} H_{\rho \sigma \tau}{ }^{M} \\
& -\frac{1}{2} \partial^{\mu} \phi^{A} \partial_{\mu} \phi^{A}-\frac{1}{2} \partial_{y} \phi^{A} \partial_{y} \phi^{A}
\end{aligned}
$$

with indices ranging along

$$
M=1, \ldots, 27, \quad A=1, \ldots, 42 .
$$

After dimensional reduction to five dimensions, this Lagrangian coincides with the Lagrangian (3.12) of linearized maximal supergravity. The Lagrangian (4.9) describes the full six-dimensional theory, with the field content of fivedimensional maximal supergravity enhanced by the fields $\widehat{C}_{\mu \nu, \rho}, \mathscr{C}_{\mu \nu, \rho}$, and $T_{\mu \nu, \rho \sigma} . D=6$ Poincaré invariance is no longer manifest although it can still be realized on the equations of motion.

\section{PROGRESS TOWARD AN EXCEPTIONAL MASTER ACTION}

In the previous sections, we have constructed Lagrangians (3.6), (4.4), and (4.9), for the three six-dimensional models which share a number of universal features and structures. In particular, after dimensional reduction to five dimensions they all reduce to the same Lagrangian (3.12) corresponding to linearized maximal supergravity in five dimensions. The three distinct six-dimensional theories are then described as different extensions of this Lagrangian by terms carrying derivatives along the sixth dimension. In the various matter sectors, these terms ensure covariantization under nontrivial gauge structures and provide sources to the field equations of five-dimensional supergravity.

This reformulation within a common framework is very much in the spirit of exceptional field theories. In that framework, higher-dimensional supergravity theories are reformulated in terms of the field content of a lowerdimensional supergravity keeping the dependence on all coordinates. More precisely, their formulation is based on a split of coordinates into $D$ external and $n$ internal coordinates of which the latter are formally embedded into a fundamental representation $\mathcal{R}_{v}$ of the global symmetry group $\mathrm{E}_{11-D,(11-D)}$ of $D$-dimensional maximal supergravity. Different embeddings of the internal coordinates into $\mathcal{R}_{v}$ then correspond to different higher-dimensional origins. Here, we will discuss a similar uniform description of the six-dimensional models based on $D=5$ external dimensions which encompasses the three different models upon proper identification of the sixth coordinate within the internal coordinates. As discussed in the Introduction this will require an enhancement of the internal coordinates of exceptional field theory by an additional exotic coordinate related to the singlet central charge in the $D=5$ supersymmetry algebra.

\section{A. Linearized ExFT and embedding of the $\mathcal{N}=(2,2)$ model}

The theory relevant for our discussion is $\mathrm{E}_{6(6)}$ exceptional field theory $[10,11]$. Its bosonic field content is given by a graviton $g_{\mu \nu}$ together with 27 vector fields $\mathcal{A}_{\mu}{ }^{M}$ and their dual tensors $\mathcal{B}_{\mu \nu M}$, together with 42 scalars parametrizing the internal metric $\mathcal{M}_{M N}=\left(\mathcal{V}^{T}\right)_{M N}$ with $\mathcal{V}$ a representative of the coset space $\mathrm{E}_{6(6)} / \mathrm{USp}(8)$. Fields depend on 5 external and 27 internal coordinates with the latter transforming in the fundamental 27 of $\mathrm{E}_{6(6)}$ and with internal coordinate dependence of the fields restricted by the section constraint [9]

$$
d^{K M N} \partial_{M} \otimes \partial_{N}=0,
$$

with the two differential operators acting on any couple of fields and gauge parameters of the theory. The tensor $d^{K M N}$ denotes the cubic totally symmetric $\mathrm{E}_{6(6)}$ invariant tensor, which we normalize as $d^{M N P} d_{M N Q}=\delta_{Q}{ }^{P}$. The section 
condition (5.1) admits two inequivalent solutions [10] which reduce the internal coordinate dependence of all fields to the 6 internal coordinates from $D=11$ supergravity, or 5 internal coordinates from IIB supergravity, respectively. For details of the ExFT Lagrangian we refer to $[10,11]$. Here, we spell out its "free" limit, obtained by linearizing the full theory according to

$$
g_{\mu \nu}=\eta_{\mu \nu}+h_{\mu \nu}, \quad \mathcal{M}_{M N}=\Delta_{M N}+\phi_{M N},
$$

around the constant background given by the Minkowski metric $\eta_{\mu \nu}$ and the identity matrix $\Delta_{M N}$. The scalar fluctuations $\phi_{M N}$ are further constrained by the coset properties of $\mathcal{M}_{M N}$. To quadratic order in the fluctuations, the ExFT Lagrangian then yields

$$
\begin{aligned}
\mathcal{L}_{\mathrm{ExFT}, \text { free }}= & -\frac{1}{4} \Omega^{\mu \nu \rho} \Omega_{\mu \nu \rho}+\frac{1}{2} \Omega^{\mu \nu \rho} \Omega_{\nu \rho \mu}+\Omega^{\mu} \Omega_{\mu} \\
& -\frac{1}{4} \mathcal{F}^{\mu \nu M} \mathcal{F}_{\mu \nu}{ }^{N} \Delta_{M N} \\
& -\frac{5}{4} \sqrt{10} \varepsilon^{\mu \nu \rho \sigma \tau} d^{M N K} \partial_{\mu} \mathcal{B}_{\nu \rho M} \partial_{N} \mathcal{B}_{\sigma \tau K} \\
& -\frac{1}{24} D_{\mu} \phi^{M N} D^{\mu} \phi_{M N}+\mathcal{L}_{\mathrm{pot}},
\end{aligned}
$$

with indices $M, N$ raised and lowered by $\Delta_{M N}$ and its inverse, and with the various elements of (5.3) given by

$$
\begin{aligned}
\Omega_{\mu \nu \rho}= & \partial_{[\mu} h_{\nu] \rho}-\frac{2}{3} \partial_{M} \mathcal{A}_{[\mu}{ }^{M} \eta_{\nu] \rho}, \quad \Omega_{\mu} \equiv \Omega_{\mu \nu}{ }^{\nu}, \\
\mathcal{F}_{\mu \nu}{ }^{M}= & 2 \partial_{[\mu} \mathcal{A}_{\nu]}{ }^{M}+10 d^{M N K} \partial_{N} \mathcal{B}_{\mu \nu K}, \\
D_{\mu} \phi^{M N}= & \partial_{\mu} \phi^{M N}+2 \partial_{K} \mathcal{A}_{\mu}{ }^{(M} \Delta^{N) K}+\frac{2}{3} \partial_{K} \mathcal{A}_{\mu}{ }^{K} \Delta^{M N}-20 \partial_{K} \mathcal{A}_{\mu}{ }^{L} d_{P L R} d^{R K(M} \Delta^{N) P}, \\
\mathcal{L}_{\mathrm{pot}}= & -\frac{1}{24} \Delta^{M N} \partial_{M} \phi^{K L} \partial_{N} \phi_{K L}+\frac{1}{2} \Delta^{M N} \partial_{M} \phi^{K L} \partial_{L} \phi_{N K}-\frac{1}{2} \partial_{M} h_{\nu}{ }^{\nu} \partial_{N} \phi^{M N} \\
& +\frac{1}{4} \Delta^{M N} \partial_{M} h_{\mu}{ }^{\mu} \partial_{N} h_{\nu}{ }^{\nu}-\frac{1}{4} \Delta^{M N} \partial_{M} h^{\mu \nu} \partial_{N} h_{\mu \nu} .
\end{aligned}
$$

The Lagrangian we have presented above for the sixdimensional $\mathcal{N}=(2,2)$ model naturally fits into this framework. This does not come as a surprise since the six-dimensional model is nothing but linearized maximal supergravity known to be described by $\mathrm{E}_{6(6)}$ ExFT upon proper selection of the sixth coordinate among the internal $\partial_{M}$. This choice is uniquely fixed by the requirement that the resulting theory exhibits the global $\mathrm{SO}(5,5)$ symmetry group of maximal six-dimensional supergravity, thus breaking

$$
\begin{aligned}
\mathrm{E}_{6(6)} \rightarrow \mathrm{SO}(5,5), \quad 27 & \rightarrow 1 \oplus 16 \oplus 10, \\
\left\{\partial_{M}\right\} & \rightarrow\left\{\partial_{0}, \partial_{i}, \partial_{a}\right\},
\end{aligned}
$$

and keeping only coordinate-dependence along the $\mathrm{SO}(5,5)$ singlet. In this split, the $\mathrm{E}_{6(6)}$ invariant symmetric tensor $d^{M N K}$ has the nonvanishing components

$$
d^{0 a b}=\frac{1}{\sqrt{10}} \eta^{a b}, \quad d^{a i j}=\frac{1}{2 \sqrt{5}}\left(\Gamma^{a}\right)^{i j},
$$

in terms of $\mathrm{SO}(5,5) \Gamma$ matrices and its invariant tensor $\eta^{a b}$ of signature $(5,5)$, showing that the section constraint $(5.1)$ is trivially satisfied as $\partial_{i}=0=\partial_{a}$. Putting this together with the linearized ExFT Lagrangian (5.3), and splitting fields as

$$
\left\{\mathcal{A}_{\mu}{ }^{M}\right\}=\left\{A_{\mu}, A_{\mu}{ }^{i}, A_{\mu}{ }^{a}\right\}, \quad \text { etc. },
$$

we arrive at

$$
\begin{aligned}
\mathcal{L}_{(2,2)}= & -\frac{1}{4} \Omega^{\mu \nu \rho} \Omega_{\mu \nu \rho}+\frac{1}{2} \Omega^{\mu \nu \rho} \Omega_{\nu \rho \mu}+\Omega^{\mu} \Omega_{\mu}-\frac{1}{4} F^{\mu \nu} F_{\mu \nu}-\frac{1}{4} F^{\mu \nu i} F_{\mu \nu}{ }^{i} \\
& -\frac{1}{4}\left(F_{\mu \nu}{ }^{a}+\partial_{y} B_{\mu \nu}{ }^{a}\right)\left(F^{\mu \nu a}+\partial_{y} B^{\mu \nu a}\right)-\frac{1}{24} \varepsilon^{\mu \nu \rho \sigma \tau} \eta_{a b} \partial_{y} B_{\mu \nu}{ }^{a} H_{\rho \sigma \tau}{ }^{b}-\frac{1}{2} \partial^{\mu} \phi^{\alpha} \partial_{\mu} \phi^{\alpha} \\
& -\frac{1}{2}\left(\partial^{\mu} \phi-\sqrt{\frac{8}{3}} \partial_{y} A^{\mu}\right)\left(\partial_{\mu} \phi-\sqrt{\frac{8}{3}} \partial_{y} A_{\mu}\right)-\frac{1}{2}\left(\partial^{\mu} \phi^{i}-\partial_{y} A^{\mu i}\right)\left(\partial_{\mu} \phi^{i}-\partial_{y} A_{\mu}{ }^{i}\right), \\
& -\frac{1}{2} \partial_{y} \phi^{\alpha} \partial_{y} \phi^{\alpha}+\frac{5}{6} \partial_{y} \phi \partial_{y} \phi-\frac{2}{3} \partial_{y} h_{\sigma}{ }^{\sigma} \partial_{y} \phi+\frac{1}{4} \partial_{y} h_{\sigma}{ }^{\sigma} \partial_{y} h_{\rho}{ }^{\rho}-\frac{1}{4} \partial_{y} h^{\mu \nu} \partial_{y} h_{\mu \nu},
\end{aligned}
$$


which precisely produces the sum of Lagrangians (3.6), (3.9), (3.11), after proper rescaling of the singlet scalar field $\phi$. The nontrivial checks of this coincidence include all the coefficients in the various connection terms, as well as in the Stïckelberg-type couplings between vector and tensor fields, and the coefficients in front of the various $\partial_{y} \phi \partial_{y} \phi$ terms in the last line. Again, this is not a surprise but a consequence of the proven equivalence of ExFT with higher-dimensional maximal supergravity. Note that although the free theory only exhibits a compact USp(4) $\times \mathrm{USp}(4)$ global symmetry, the couplings exhibited in (5.8) are far more constrained than allowed by this symmetry and witness the underlying $\mathrm{E}_{6(6)}$ structure broken to $\mathrm{SO}(5,5)$ according to (5.5), (5.6).

The ExFT Lagrangian is to a large extent determined by invariance under generalized internal diffeomorphisms acting with a gauge parameter $\Lambda^{M}$ in the fundamental 27. After linearization (5.2) these diffeomorphisms act as

$$
\begin{aligned}
\delta \phi_{M N}= & 2 \Delta_{K(M} \partial_{N)} \Lambda^{K}+\frac{2}{3} \partial_{K} \Lambda^{K} \Delta_{M N} \\
& -20 d^{P K R} d_{R L(M} \Delta_{N) P} \partial_{K} \Lambda^{L}, \\
\delta \mathcal{A}_{\mu}{ }^{M}= & \partial_{\mu} \Lambda^{M}, \quad \delta h_{\mu \nu}=\frac{2}{3} \partial_{M} \Lambda^{M} \eta_{\mu \nu},
\end{aligned}
$$

and one can show invariance of the linearized Lagrangian (5.8), provided the section constraint (5.1) is satisfied.

\section{B. Beyond standard ExFT: Embedding of the $\mathcal{N}=(3,1)$ and $(4,0)$ couplings}

As we have discussed in the Introduction, the charges carried by the massive BPS multiplets in the reduction of the $\mathcal{N}=(3,1)$ and the $\mathcal{N}=(4,0)$ model, respectively, suggest that an inclusion of these models into the framework of ExFT necessitates an extension of the space of 27 internal coordinates by an additional exotic coordinate corresponding to the singlet central charge [7]. Denoting derivatives along this coordinate by $\partial_{0}$, this would amount to a relaxation of the standard section constraint (5.1) to a constraint of the form

$d^{K M N} \partial_{M} \otimes \partial_{N}-\frac{1}{\sqrt{10}} \Delta^{K M}\left(\partial_{M} \otimes \partial_{\bullet}+\partial_{\bullet} \otimes \partial_{M}\right)=0$,

which at the present stage only makes sense in the linearized theory where $\Delta^{K M}$ is a constant background tensor. Apart from the standard ExFT solutions

$$
d^{K M N} \partial_{M} \otimes \partial_{N}=0, \quad \partial_{\bullet}=0,
$$

of this constraint, which allow the embedding of the $\mathcal{N}=$ $(2,2)$ model as described above, the extended section constraint also allows for two exotic solutions

$$
\begin{aligned}
(3,1): \partial_{y}^{(3,1)}= & \frac{2}{\sqrt{3}} \partial_{0}=-2 \partial . \\
& \text { with the } \mathrm{F}_{4(4)} \text { singlet } \partial_{0} \subset \partial_{M}, \\
(4,0): \partial_{y}^{(4,0)}= & -\partial_{.}, \quad \partial_{M}=0,
\end{aligned}
$$

corresponding to the two exotic six-dimensional models in precise correspondence with the central charges carried by the corresponding BPS multiplets [7]. While the $(4,0)$ solution trivially solves the constraint (5.10), the $\mathcal{N}=$ $(3,1)$ solution is based on the decomposition

$$
\begin{aligned}
\mathrm{E}_{6(6)} \rightarrow \mathrm{F}_{4(4)}, \quad 27 & \rightarrow 1 \oplus 26, \\
\left\{\partial_{M}\right\} & \rightarrow\left\{\partial_{0}, \partial_{A}\right\},
\end{aligned}
$$

under which the symmetric $d$-tensor decomposes into

$d^{000}=-\frac{2}{\sqrt{30}}, \quad d^{0 A B}=\frac{1}{\sqrt{30}} \eta^{A B}, \quad d^{A B C}$,

with the $\mathrm{F}_{4(4)}$ invariant symmetric tensor $\eta^{A B}$ of signature $(14,12)$, and the symmetric invariant tensor $d^{A B C}$ satisfying

$$
d^{A B C} \eta_{B C}=0, \quad d_{A B C} d^{A B D}=\frac{14}{15} \delta_{C}^{D} .
$$

This shows explicitly how the $(3,1)$ assignment of $(5.12)$ also provides a solution to the extended section constraint (5.10).

It is intriguing to study the fate of diffeomorphism invariance of the ExFT Lagrangian (5.3) if the original section constraint is relaxed to (5.10). Except for the last term in (5.3), the Lagrangian remains manifestly invariant without any use of the section constraint. Explicit variation of the potential term $\mathcal{L}_{\text {pot }}$ under linearized diffeomorphisms (5.9) on the other hand yields (up to total derivatives)

$$
\begin{aligned}
\delta_{\Lambda} \mathcal{L}_{\mathrm{pot}}= & \left(5 \Delta_{L S} d^{L M N} d^{K P Q}-10 \Delta^{M N} \Delta^{K L} d_{L S R} d^{R P Q}\right) \\
& \times \Lambda^{S} \partial_{P} \partial_{Q} \partial_{M} \phi_{N K} \\
& -10 h_{\mu}{ }^{\mu} \Delta^{M K} d_{K L R} d^{R P Q} \partial_{M} \partial_{P} \partial_{Q} \Lambda^{L}
\end{aligned}
$$

which consistently vanishes modulo the standard section constraint (5.1). For the weaker constraint (5.10), this variation no longer vanishes and may be recast in the following form:

$\delta_{\Lambda} \mathcal{L}_{\text {pot }}=\Delta^{K M} \Lambda^{N} \partial . \partial . \partial_{M} \phi_{N K}-4 h_{\nu}{ }^{\nu} \partial . \partial . \partial_{N} \Lambda^{N}$,

after repeated use of (5.10) and further manipulation of the expressions. In order to compensate for this variation let us first note that there is no possible covariant extension of the transformation rules (5.10) by terms carrying $\partial . \Lambda^{M}$, such that invariance can only be restored by extending the potential. A possible such extension is given by 


$$
\begin{aligned}
\mathcal{L}_{\mathrm{pot}, \bullet}= & \mathcal{L}_{\mathrm{pot}}-\frac{1}{24} \partial . \phi_{M N} \partial . \phi^{M N}-\frac{3}{4} \partial \cdot h_{\sigma}{ }^{\sigma} \partial \cdot h_{\rho}{ }^{\rho} \\
& +\frac{3}{4} \partial \cdot h^{\mu \nu} \partial \cdot h_{\mu \nu},
\end{aligned}
$$

and it is straightforward to verify that the variation of the additional terms in (5.18) precisely cancels the contributions in (5.17), such that

$$
\delta_{\Lambda} \mathcal{L}_{\text {pot, }}=0
$$

For the exotic solutions of the section constraint, the $\partial . \phi_{M N} \partial . \phi^{M N}$ terms in (5.18) give rise to additional contributions of the type $\partial_{y} \phi \partial_{y} \phi$ in the Lagrangian. Collecting all such terms in (5.18) for the two exotic solutions (5.12) yields

$$
\begin{aligned}
(3,1) \rightarrow-\frac{1}{2} \partial_{y} \phi^{\alpha} \partial_{y} \phi^{\alpha}, & \alpha=1, \ldots, 28, \\
(4,0) \rightarrow-\frac{1}{2} \partial_{y} \phi^{A} \partial_{y} \phi^{A}, & A=1, \ldots, 42 .
\end{aligned}
$$

These are precisely the terms found in our explicit construction of actions (4.4) and (4.9) above. In other words, the relaxation (5.10) of the section constraint together with generalized diffeomorphism invariance precisely implies the correct scalar couplings in the Lagrangians of the exotic models. In addition, the $\partial . h \partial . h$ terms in (5.18) cancel the corresponding terms in $\mathcal{L}_{\text {pot }}(5.4)$ upon selecting the $(3,1)$ solution of the section constraint (5.12), just as required in order to reproduce the correct Lagrangian of the $\mathcal{N}=(3,1)$ model (4.2). ${ }^{4}$

We may continue the symmetry analysis for the tensor gauge transformations given by a gauge parameter $\Lambda_{\mu M}$ in standard ExFT. For these transformations there is a natural extension of the standard ExFT transformation rules in the presence of the exotic coordinate and exotic fields as

$$
\begin{aligned}
\delta_{\Lambda_{\mu}} \mathcal{A}_{\mu}{ }^{M} & =-10 d^{M N K} \partial_{N} \Lambda_{\mu K}-\sqrt{10} \Delta^{M K} \partial . \Lambda_{\mu K}, \\
\delta_{\Lambda_{\mu}} \mathcal{B}_{\mu \nu M} & =2 \partial_{[\mu} \Lambda_{\nu] M} .
\end{aligned}
$$

Computing the action of these transformations on the connection featuring in the covariant scalar derivatives $D_{\mu} \phi^{M N}$ in (5.4), we obtain after some manipulation ${ }^{5}$

$$
\begin{aligned}
\delta_{\Lambda_{\mu}} D_{\mu} \phi^{M N}= & 10\left(\frac{1}{3} \Delta^{M N} \delta_{P} Q+\Delta^{Q(M} \delta_{P}^{N)}-10 \Delta^{S(M} d^{N) Q R} d_{R S P}\right) d^{P K L} \partial_{K} \partial_{L} \Lambda_{\mu Q} \\
& -2 \sqrt{10}\left(\frac{1}{3} \Delta^{M N} \delta_{P} Q+\delta_{P}{ }^{(M} \Delta^{N) Q}-10 \Delta^{L(M} d^{N) Q R} d_{R P L}\right) \Delta^{P K} \partial_{K} \partial . \Lambda_{\mu Q} .
\end{aligned}
$$

The resulting expression precisely vanishes with the modified section constraint (5.10). This shows the necessity of the $\partial . \Lambda_{\mu M}$ terms in (5.21) in order to maintain gauge invariance of the kinetic term $D_{\mu} \phi^{M N} D^{\mu} \phi_{M N}$ in presence of the relaxed section constraint. It is straightforward to verify that these additional terms in the transformation induce a modification of the gauge invariant vector field strengths to

$$
\mathcal{F}_{\mu \nu}{ }^{M} \equiv 2 \partial_{[\mu} \mathcal{A}_{\nu]}{ }^{M}+10 d^{M N K} \partial_{N} \mathcal{B}_{\mu \nu K}+\sqrt{10} \Delta^{M K} \partial . \mathcal{B}_{\mu \nu K},
$$

as well an extension of the topological term, such that the combined vector-tensor couplings take the form

$$
\mathcal{L}_{\mathrm{vt}, \bullet}=-\frac{1}{4} \Delta_{M N} \mathcal{F}^{\mu \nu M} \mathcal{F}_{\mu \nu}{ }^{N}-\frac{5}{4} \varepsilon^{\mu \nu \rho \sigma \tau} \partial_{\mu} \mathcal{B}_{\nu \rho M}\left(\sqrt{10} d^{M N K} \partial_{N} \mathcal{B}_{\sigma \tau K}+\Delta^{M K} \partial . \mathcal{B}_{\sigma \tau K}\right),
$$

and are invariant under these gauge transformations. Let us work out the effect of these modifications for the exotic solutions of the section constraint. With the kinetic scalar term unchanged, the resulting couplings are directly inferred from evaluating the covariant derivatives (5.4) for the $d$-symbol (5.14), giving rise to

$$
\begin{aligned}
& (3,1) \longrightarrow-\frac{1}{2}\left(\partial^{\mu} \phi^{i}-\partial_{y} A^{\mu i}\right)\left(\partial_{\mu} \phi^{i}-\partial_{y} A_{\mu}{ }^{i}\right)-\frac{1}{2} \partial^{\mu} \phi^{\alpha} \partial_{\mu} \phi^{\alpha}, \quad i=1, \ldots, 14, \alpha=1, \ldots, 28, \\
& (4,0) \longrightarrow-\frac{1}{2} \partial^{\mu} \phi^{A} \partial_{\mu} \phi^{A}, \quad A=1, \ldots, 42
\end{aligned}
$$

\footnotetext{
${ }^{4}$ In contrast, these terms appear in conflict with embedding the spin-2 sector of the $\mathcal{N}=(4,0)$ model as they survive under the $(4,0)$ solution in (5.12) but should be absent in the final Lagrangian (4.6). We come back to this in Sec. V C.

${ }^{5} \mathrm{~A}$ useful identity for this computation is given by

$$
d^{P L Q} d_{P S R} d^{K M R} \partial_{K} \partial_{L}=\frac{1}{10} \delta_{S}^{K} d^{L Q M} \partial_{K} \partial_{L}+\frac{1}{20} \delta_{S}^{M} d^{Q K L} \partial_{K} \partial_{L}+\frac{1}{20} \delta_{S}^{Q} d^{M K L} \partial_{K} \partial_{L}-\frac{1}{2} d^{Q M R} d_{R S P} d^{P K L} \partial_{K} \partial_{L},
$$

generalizing Eqs. (2.12), (2.13) of [11].
} 
This precisely reproduces the vector-scalar couplings found in the explicit Lagrangians (4.4), (4.9) above. As for the vectortensor couplings, evaluating the Lagrangian (5.24) with (5.14) for the solutions (5.12) gives rise to the explicit couplings

$$
\begin{aligned}
(3,1) \longrightarrow & -\frac{1}{4}\left(F_{\mu \nu}+\frac{3 \sqrt{3}}{2} \partial_{y} B_{\mu \nu}\right)\left(F^{\mu \nu}+\frac{3 \sqrt{3}}{2} \partial_{y} B^{\mu \nu}\right)-\frac{1}{4}\left(F_{\mu \nu}{ }^{a}+\partial_{y} B_{\mu \nu}{ }^{a}\right)\left(F^{\mu \nu a}+\partial_{y} B^{\mu \nu a}\right) \\
& -\frac{1}{4} F^{\mu \nu i} F_{\mu \nu}{ }^{i}-\frac{3}{16} \varepsilon^{\mu \nu \rho \sigma \tau} \partial_{y} B_{\mu \nu} H_{\rho \sigma \tau}-\frac{1}{24} \varepsilon^{\mu \nu \rho \sigma \tau} \partial_{y} B_{\mu \nu}{ }^{a} H_{\rho \sigma \tau}{ }^{a}, \\
(4,0) \longrightarrow & -\frac{1}{4}\left(F_{\mu \nu}{ }^{M}+\partial_{y} B_{\mu \nu}{ }^{M}\right)\left(F^{\mu \nu M}+\partial_{y} B^{\mu \nu M}\right)-\frac{1}{24} \varepsilon^{\mu \nu \rho \sigma \tau} \partial_{y} B_{\mu \nu}{ }^{M} H_{\rho \sigma \tau}{ }^{M},
\end{aligned}
$$

with indices in range $i=1, \ldots, 14, \quad a=1, \ldots, 12$, $M=1, \ldots, 27$, as above. Again, this precisely reproduces the couplings found above (after proper rescaling of the vector field $A_{\mu}$ ).

To summarize, in the scalar, vector, and tensor sector, we have constructed an extension of the ExFT Lagrangian (at the linearized level), given by

$$
\mathcal{L}=-\frac{1}{2} D_{\mu} \phi^{M N} D^{\mu} \phi_{M N}+\mathcal{L}_{\mathrm{vt}, \bullet}+\mathcal{L}_{\mathrm{pot}, \bullet},
$$

which is invariant under the gauge transformations (5.9), (5.21) modulo the relaxed section constraint (5.10). The weaker section constraint necessitates a number of additional contributions to the Lagrangian (and transformation rules) which precisely reproduce the explicit couplings found in the Lagrangians of the exotic models (4.4), (4.9) constructed above. It is remarkable that this match confirms the couplings that have been determined from an underlying noncompact $\mathrm{E}_{6(6)}$ and $\mathrm{F}_{4(4)}$ structure, respectively, despite the fact that the free theory only exhibits invariance under the compact $R$-symmetry subgroup $\operatorname{USp}\left(2 \mathcal{N}_{+}\right) \times$ $\operatorname{USp}\left(2 \mathcal{N}_{-}\right)$which might in principle allow for much more general couplings. We take this as evidence for the conjectured $\mathrm{E}_{6(6)}$ and $\mathrm{F}_{4(4)}$ invariance of the putative interacting theories [1].

\section{The spin-2 sector}

The above findings have revealed a very intriguing common structure of the couplings in the scalar, vector, and tensor sectors of the different models which can be consistently embedded into an extension of (linearized) exceptional field theory. For the spin-2 sector carrying the Pauli-Fierz field and its duals on the other hand the picture appears not yet complete. Extrapolation of the Lagrangian of the $\mathcal{N}=(4,0)$ model (4.6) suggests an extension of the standard ExFT Lagrangian by couplings carrying $\partial$. derivatives and the dual graviton fields as

$$
\begin{aligned}
& \mathcal{L}=-\frac{1}{4} \widehat{\Omega}^{\mu \nu \rho} \widehat{\Omega}_{\mu \nu \rho}+\frac{1}{2} \widehat{\Omega}^{\mu \nu \rho} \widehat{\Omega}_{\nu \rho \mu}+\widehat{\Omega}^{\mu} \widehat{\Omega}_{\mu}+\frac{1}{8} \varepsilon_{\mu \nu \sigma \kappa \lambda} \partial^{\mu} \widehat{C}_{\rho}^{\nu \sigma} \partial . \widehat{C}^{\kappa \lambda, \rho} \\
& -\frac{1}{32} \varepsilon_{\mu \nu \sigma \kappa \lambda} \partial^{\mu} \mathscr{C}_{\rho}^{\nu \sigma} \partial . \mathscr{C}^{\kappa \lambda, \rho}+\frac{1}{8} \partial . \mathscr{C}_{\sigma \tau, \nu} \partial_{\mu} T^{\mu \nu, \sigma \tau}-\frac{1}{4} \partial . \mathscr{C}_{\kappa \lambda, \tau} \partial^{\kappa} T^{\lambda \sigma, \tau}{ }_{\sigma} \\
& -\frac{1}{4} \partial_{\nu} \mathscr{C}_{\sigma \mu}{ }^{\mu} \partial . T^{\sigma \tau, \nu}{ }_{\tau}+\frac{1}{8} \partial . \mathscr{C}_{\sigma \mu}{ }^{\mu} \partial^{\sigma} T_{\tau \nu}{ }^{\tau \nu}+\frac{1}{64} \varepsilon_{\mu \nu \alpha \beta \gamma} \partial^{\alpha} T_{\sigma \tau}{ }^{\beta \gamma} \partial . T^{\mu \nu, \sigma \tau} \\
& -\frac{1}{32} \partial . T_{\sigma \tau, \mu \nu} \partial . T^{\mu \nu, \sigma \tau}+\frac{1}{8} \partial . T_{\sigma \mu, \nu}{ }^{\mu} \partial . T^{\sigma \tau, \nu}{ }_{\tau}-\frac{1}{32} \partial . T_{\mu \nu}{ }^{\mu \nu} \partial . T_{\sigma \tau}{ }^{\sigma \tau} \\
& +\frac{5}{4} \varepsilon^{\mu \nu \rho \sigma \tau} d^{K M N} \partial_{K} \mathcal{B}_{\mu \nu M} \partial_{N} \widehat{C}_{\rho \sigma, \tau}
\end{aligned}
$$

with

$\widehat{\Omega}_{\mu \nu \rho}=\partial_{[\mu} h_{\nu] \rho}-\frac{2}{3} \partial_{M} A_{[\mu}{ }^{M} \eta_{\nu] \rho}-\partial . \widehat{C}_{\mu \nu, \rho}+\frac{1}{2} \partial . \mathscr{C}_{\mu \nu, \rho}$.

By construction, this reproduces the $\mathcal{N}=(2,2)$ and the $\mathcal{N}=(4,0)$ models upon choosing the corresponding solutions of the section constraint. It remains unclear however, how the spin-2 sector of the $\mathcal{N}=(3,1)$ model can find its place in this construction. In particular, the appearance of the extra fields $\mathscr{C}_{\mu \nu, \rho}$ and $T_{\mu \nu, \rho \sigma}$ appearing in (5.28), whose couplings remain present upon selecting the $(3,1)$ solution $(5.12)$ of the section constraint, poses a challenge for recovering the Lagrangian (4.4) of the $\mathcal{N}=$ $(3,1)$ model. The structure of the gauge transformations of $\mathscr{C}$ as extrapolated from (4.8) appears to suggest a gauge fixing of the $\zeta_{\mu \nu}$ and $\lambda_{\rho, \mu \nu}$ gauge symmetries - absent in the $\mathcal{N}=(3,1)$ model - in order to remove this field. Another apparent problem in the spin-2 sector is the lacking 
reconciliation between the $\partial . h \partial . h$ terms from (5.18) and the $\partial . T \partial . T$ terms of (5.28) which mutually violate the correct limits to the exotic models. Resolution of this problem may require to implement algebraic relations between the Pauli-Fierz $h_{\mu \nu}$ field and the double dual graviton [2] (see also [27]).

\section{CONCLUSIONS AND OUTLOOK}

In this paper we have taken the first step in constructing action principles for exotic supergravity theories in $6 \mathrm{D}$ by giving such actions for the free bosonic part. These actions show already intriguing new features such as the simultaneous appearance of (linearized) diffeomorphisms and dual diffeomorphisms, which are realized on exotic Young tableaux fields as well as more conventional gravity fields. Our formulation abandons manifest 6D Lorentz invariance, as expected to be necessary on general grounds, by being based on a $5+1$ split of coordinates. Remarkably, the field equations implied by our actions can be integrated to reconstruct the correct dynamics of these exotic supergravites. Moreover, we have seen the first glimpses of an exceptional field theory master formulation, in which the conventional $\mathcal{N}=(2,2)$, as well as the exotic $\mathcal{N}=(3,1)$ and $\mathcal{N}=(4,0)$ models all emerge through different solutions of an extended section constraint, but clearly much more needs to be done. We close with a brief discussion of possible future developments.

First, it remains to exhibit the (maximal) supersymmetries in these nonstandard formulations, even just at the free level. We have no doubt that this can be achieved as in exceptional field theory where different supersymmetries (such as type IIB versus type IIA) are realized within a single master formulation. Second, it would be interesting to study possible embeddings into exceptional field theories of higher rank, such as for U-duality groups $\mathrm{E}_{7(7)}$ and $\mathrm{E}_{8(8)}$, which may illuminate some issues and which can also be done already at linearized level. Finally, the most important outstanding problem is clearly the question whether our formulation can be extended to the nonlinear interacting theory. We would like to emphasize that the present formulations seem quite promising in this regard since they feature not only the exotic fields but also the more conventional gravity fields, which come with an action that allows a natural embedding into the full nonlinear Einstein-Hilbert action. In turn this suggests that all these fields might become part of a tensor hierarchy that extends to the gravity sector. If so this could quite naturally lend itself to a formulation of nonlinear dynamics in terms of a hierarchy of duality relations as in [28].

\section{ACKNOWLEDGMENTS}

We wish to thank X. Bekaert, M. Henneaux, C. Hull, and V. Lekeu for enlightening discussions. The work of O. H. is supported by the ERC Consolidator Grant "Symmetries \& Cosmology."

Note added.-While finalizing the present paper, [29] appeared, which also investigates exotic theories in 6D.

\section{APPENDIX A: ACTIONS FOR SELF-DUAL TENSOR FIELDS}

It is well known that the first-order field equations for $D=6$ self-dual tensor fields

$$
H_{\hat{\mu} \hat{\nu} \hat{\rho}}=\frac{1}{6} \varepsilon_{\hat{\mu} \hat{\nu} \hat{\rho} \hat{\sigma} \hat{\kappa} \hat{\lambda}} H^{\hat{\sigma} \hat{\kappa} \hat{\lambda}}, \quad H_{\hat{\mu} \hat{\nu} \hat{\rho}}=3 \partial_{[\hat{\mu}} B_{\hat{\nu} \hat{\rho}]}
$$

do not integrate to a standard action principle, yet various mechanisms with different characteristics have been devised such as to provide a Lagrangian description of these equations [13,20-22]. In this Appendix, we briefly review the construction of Henneaux and Teitelboim [13] which is somewhat closest in spirit to the construction employed in this paper, together with its dual formulation that is naturally embedded within exceptional field theory. Both formulations are based on a coordinate split (3.1)

$$
\left\{x^{\hat{\mu}}\right\} \rightarrow\left\{x^{\mu}, y\right\}
$$

and sacrifice manifest $D=6$ Poincaré invariance. ${ }^{6}$ With the corresponding split $\left\{B_{\hat{\mu} \hat{\nu}}\right\}=\left\{B_{\mu \nu}, B_{\mu 5} \equiv A_{\mu}\right\}$ of the six-dimensional tensor field, the self-duality equations (A1) take the form

$\mathcal{F}_{\mu \nu}+\frac{1}{6} \varepsilon_{\mu \nu \rho \sigma \tau} H^{\rho \sigma \tau}=0, \quad$ for $\mathcal{F}_{\mu \nu} \equiv F_{\mu \nu}+\partial_{y} B_{\mu \nu}$.

In particular, the divergence and curl of this equation give rise to

$$
\begin{aligned}
\partial^{\mu} \mathcal{F}_{\mu \nu} & =0, \\
\varepsilon^{\mu \nu \lambda \sigma \tau} \partial_{y} H_{\lambda \sigma \tau}-6 \partial_{\lambda} H^{\lambda \mu \nu} & =0,
\end{aligned}
$$

respectively.

\section{Henneaux-Teitelboim Lagrangian}

The Lagrangian proposed by Henneaux and Teitelboim [13] for the description of the self-dual tensors takes the form

$$
\mathcal{L}=\frac{1}{24} \varepsilon^{\mu \nu \rho \sigma \tau} \mathcal{F}_{\mu \nu} H_{\rho \sigma \tau}-\frac{1}{12} H_{\mu \nu \rho} H^{\mu \nu \rho}
$$

\footnotetext{
${ }^{6}$ The original construction of [13] defines the $5+1$ split by singling out the time coordinate, but the method obviously applies equally well for the split based on a spatial distinguished dimension.
} 
when applied to Eq. (A3), i.e., evaluated for spacelike split and flat background. As a first observation, this Lagrangian depends on the vector field $A_{\mu}$ only via total derivatives, such that it does not show up in the field equations

$$
\varepsilon_{\mu \nu \rho \sigma \tau} \partial_{y} H^{\rho \sigma \tau}=6 \partial^{\rho} H_{\mu \nu \rho}
$$

reproducing the second equation of (A4). This equation now serves as an integrability equation in order to locally define the vector field $A_{\mu}$ via the equation

$$
2 \partial_{[\mu} A_{\nu]}=-\partial_{y} B_{\mu \nu}-\frac{1}{6} \varepsilon_{\mu \nu \rho \sigma \tau} H^{\rho \sigma \tau} .
$$

Indeed, the curl of the right-hand side vanishes by virtue of (A6). Defining the vector field $A_{\mu}$ by (A7), we precisely recover the equations of motion (A3).

\section{ExFT type Lagrangian}

Exceptional field theory typically yields formulations of higher-dimensional supergravity theories based on the field content of lower-dimensional theories. In particular, it offers actions for theories that do not admit actions in terms of their original variables, such as IIB supergravity, cf. [12]. In the context of (anti-)self-dual tensor fields appearing in six dimensions, an exceptional field theory formulation based on a split (A2) gives rise to an action

$$
\mathcal{L}=-\frac{1}{4} \mathcal{F}_{\mu \nu} \mathcal{F}^{\mu \nu}-\frac{1}{24} \varepsilon^{\mu \nu \rho \sigma \tau} \partial_{y} B_{\mu \nu} H_{\rho \sigma \tau},
$$

carrying the fields of Eq. (A3). The field equations are now given by

$$
\begin{gathered}
0=\partial^{\nu} \mathcal{F}_{\nu \mu}=\partial^{\nu} F_{\nu \mu}+\partial_{y} \partial^{\nu} B_{\nu \mu}, \\
0=\partial_{y}\left(\mathcal{F}_{\mu \nu}+\frac{1}{6} \varepsilon_{\mu \nu \rho \sigma \tau} H^{\rho \sigma \tau}\right) .
\end{gathered}
$$

In particular, Eq. (A10) implies the original field equations (A3) up to some function that does not depend on $y$ :

$$
\mathcal{F}_{\mu \nu}+\frac{1}{6} \varepsilon_{\mu \nu \rho \sigma \tau} H^{\rho \sigma \tau}=\chi_{\mu \nu}, \quad \partial_{y} \chi_{\mu \nu}=0 .
$$

Comparing the divergence of this equation to (A9), we find that locally the field $\chi_{\mu \nu}$ can be integrated to

$$
\partial^{\mu} \chi_{\mu \nu}=0 \Rightarrow \chi_{\mu \nu}=\varepsilon_{\mu \nu \rho \sigma \tau} \partial^{\rho} b^{\sigma \tau},
$$

in terms of a function $b_{\mu \nu}$, such that the field equations (A11) can be rewritten as

$$
\left(F_{\mu \nu}+\partial_{y} \tilde{B}_{\mu \nu}\right)+\frac{1}{6} \varepsilon_{\mu \nu \rho \sigma \tau} 3 \partial^{\rho} \tilde{B}^{\sigma \tau}=0,
$$

with the modified two-form

$$
\tilde{B}_{\mu \nu} \equiv B_{\mu \nu}-2 b_{\mu \nu} .
$$

In terms of the fields $A_{\mu}, \tilde{B}_{\mu \nu}$, we thus recover the desired original field equations (A3). Note finally, that the Lagrangian (A8) precisely comes with a gauge freedom of the type (A14) which allows one to absorb $b_{\mu \nu}$ into $B_{\mu \nu}$.

We thus arrive at two complementary Lagrangians (A5), (A8), which both describe the six-dimensional self-dual tensor field upon sacrificing manifest $D=6$ Poincaré invariance. They are dual to each other in the sense that upon dimensional reduction to $D=5$ dimensions, i.e., upon setting $\partial_{y} \rightarrow 0$, the Lagrangian (A8) describes the 3 degrees of freedom in terms of a free Maxwell field whereas (A5) describes them in terms of the dual massless tensor field $B_{\mu \nu}$. Similarly, the two Lagrangians (A5) and (A8) can be dualized into each other in presence of the sixth dimension.

\section{APPENDIX B: 6D FIELD EQUATIONS FROM THE NEW LAGRANGIANS}

In this Appendix, we present in detail how the secondorder field equations obtained by variation of the Lagrangians (4.2) and (4.6) can be integrated to the first-order field equations which in turn imply the original $6 \mathrm{D}$ second-order self-duality equations of the $\mathcal{N}=(3,1)$ model and the $\mathcal{N}=(4,0)$ model, respectively.

\section{The $\mathcal{N}=(3,1)$ model}

Here, we show how the second-order field equations obtained by variation of the Lagrangian (4.2) for the $\mathcal{N}=$ $(3,1)$ model can be integrated to the first-order field equations (3.21) and (3.22) which in turn imply the original 6D second-order self-duality equations (2.11).

\section{a. Field equations}

Let us first spell out the field equations obtained from variation of the Lagrangian (4.2).

Variation with respect to $A_{\mu}$ :

$$
\begin{aligned}
& \partial^{\mu} F_{\mu \nu}+\frac{3}{2} \partial_{y} \partial^{\mu} B_{\mu \nu} \\
& -\frac{1}{2} \partial_{y}\left(\partial^{\mu} h_{\mu \nu}-\partial_{\nu} h_{\mu}{ }^{\mu}-\partial_{y} \widehat{C}_{\nu \mu}{ }^{\mu}+4 \partial_{y} A_{\nu}\right)=0,
\end{aligned}
$$

which is exactly (3.29).

Variation with respect to $B_{\mu \nu}$ :

$\partial_{y}\left(F_{\mu \nu}+\frac{3}{2} \partial_{y} B_{\mu \nu}+\frac{1}{2} \varepsilon_{\mu \nu \rho \sigma \tau} \partial^{\rho} B^{\sigma \tau}+\frac{1}{12} \partial_{y} \varepsilon_{\mu \nu \rho \sigma \tau} \widehat{C}^{\rho \sigma \tau}\right)=0$, 
which is the $\partial_{y}$ derivative of Eq. (3.21).

Variation with respect to $h_{\mu \nu}$ :

$\mathcal{G}_{\mu \nu}+\frac{1}{2} \partial_{y}\left(\partial^{\rho} \widehat{C}_{\rho(\mu, \nu)}+\partial_{(\mu} \widehat{C}_{\nu) \rho}{ }^{\rho}-\eta_{\mu \nu} \partial^{\rho} \widehat{C}_{\rho \sigma}{ }^{\sigma}\right)=0$, with the linearized Einstein tensor as it appears in (3.17); this variation thus exactly reproduces the Einstein equation (3.17).

Variation with respect to $\widehat{C}_{\mu \nu, \rho}$ :

$$
\begin{aligned}
\partial_{y}\left(\partial_{[\mu} h_{\nu] \rho}+\partial^{\sigma} h_{\sigma[\mu} \eta_{\nu] \rho}-\eta_{\rho[\nu} \partial_{\mu]} h_{\sigma}{ }^{\sigma}+\frac{1}{4} \varepsilon_{\mu \nu \lambda \sigma \tau} \partial^{\lambda} \widehat{C}_{\rho}^{\sigma \tau}+\frac{1}{4} \partial_{y}\left(\widehat{C}_{\mu \nu, \rho}-\widehat{C}_{\nu \rho, \mu}-\widehat{C}_{\rho \mu, \nu}\right)\right. \\
\left.-\eta_{\rho[\nu} \partial_{y} \widehat{C}_{\mu] \sigma}{ }^{\sigma}+3 \partial_{y} A_{[\mu} \eta_{\nu] \rho}+\frac{3}{8} \varepsilon_{\mu \nu \rho \sigma \tau} B^{\sigma \tau}\right)=0,
\end{aligned}
$$

which we can further project onto its $(2,1)$ part and totally antisymmetric part

$$
\begin{gathered}
\partial_{y}\left(\partial_{[\mu} h_{\nu] \rho}+\partial^{\sigma} h_{\sigma[\mu} \eta_{\nu] \rho}-\eta_{\rho[\nu} \partial_{\mu]} h_{\sigma}{ }^{\sigma}+\frac{1}{4} \varepsilon_{\mu \nu \lambda \sigma \tau} \partial^{\lambda} \widehat{C}_{\rho}^{\sigma \tau}-\frac{1}{4} \varepsilon_{\lambda \sigma \tau[\mu \nu} \partial^{\lambda} \widehat{C}_{\rho]}^{\sigma \tau}{ }_{\rho]}\right. \\
\left.+\frac{1}{4} \partial_{y}\left(\widehat{C}_{\mu \nu, \rho}-\widehat{C}_{\nu \rho, \mu}-\widehat{C}_{\rho \mu, \nu}+\widehat{C}_{[\mu \nu, \rho]}\right)-\eta_{\rho[\nu} \partial_{y} \widehat{C}_{\mu] \sigma}{ }^{\sigma}+3 \partial_{y} A_{[\mu} \eta_{\nu] \rho}\right)=0, \\
\partial_{y}\left(\frac{1}{4} \varepsilon_{\lambda \sigma \tau[\mu \nu} \partial^{\lambda} \widehat{C}_{\rho]}^{\sigma \tau}-\frac{1}{4} \partial_{y} \widehat{C}_{[\mu \nu, \rho]}+\frac{3}{8} \partial_{y} \varepsilon_{\mu \nu \rho \sigma \tau} B^{\sigma \tau}\right)=0 .
\end{gathered}
$$

We now show how to recover the full 6D system (3.19) and (3.21) from the equations derived above. Let us first rewrite these equations in terms of the original fields of the $(3,1)$ model and integrate all the equations under $\partial_{y}$ by introducing three functions $\chi_{\mu \nu}\left(x^{\mu}\right), \psi_{\mu \nu \rho}\left(x^{\mu}\right)$ and $\varphi_{\mu \nu, \rho}\left(x^{\mu}\right)$ which are respectively antisymmetric, antisymmetric and of $(2,1)$ type, and do not depend on the sixth coordinate:

$$
\begin{gathered}
\partial^{\mu} F_{\mu \nu}+\frac{3}{2} \partial_{y} \partial^{\mu} B_{\mu \nu} \\
-\frac{1}{2} \partial_{y}\left(\partial^{\mu} h_{\mu \nu}-\partial_{\nu} h_{\mu}{ }^{\mu}-\partial_{y} C_{\nu \mu}{ }^{\mu}+4 \partial_{y} A_{\nu}\right)=0, \\
F_{\mu \nu}+\frac{3}{2} \partial_{y} B_{\mu \nu}-\partial_{y} u_{\mu \nu}+\frac{1}{2} \varepsilon_{\mu \nu \rho \sigma \tau} \partial^{\rho} B^{\sigma \tau}=\chi_{\mu \nu}, \\
\varepsilon_{\lambda \sigma \tau[\mu \nu} \partial^{\lambda} C_{\rho]}^{\sigma \tau}-4 \partial_{[\rho} u_{\mu \nu]}-\partial_{y} \varepsilon_{\mu \nu \rho \sigma \tau}\left(u^{\sigma \tau}-\frac{3}{2} B^{\sigma \tau}\right)=\psi_{\mu \nu \rho},
\end{gathered}
$$

$\mathcal{G}_{\mu \nu}+\frac{1}{2} \partial_{y}\left(\partial^{\rho} C_{\rho(\mu, \nu)}+\partial_{(\mu} C_{\nu) \rho}{ }^{\rho}-\eta_{\mu \nu} \partial^{\rho} C_{\rho \sigma}^{\sigma}\right)=0$,

$$
\begin{aligned}
& \partial_{[\mu} h_{\nu] \rho}+\partial^{\sigma} h_{\sigma[\mu} \eta_{\nu] \rho}-\eta_{\rho[\nu} \partial_{\mu]} h_{\sigma}{ }^{\sigma}+\frac{1}{4} \varepsilon_{\mu \nu \lambda \sigma \tau} \partial^{\lambda} C_{\rho}^{\sigma \tau} \\
& -\frac{1}{4} \varepsilon_{\lambda \sigma \tau[\mu \nu} \partial^{\lambda} C_{\rho]}^{\sigma \tau}+\frac{1}{2} \partial_{y} C_{\mu \nu, \rho}-\eta_{\rho[\nu} \partial_{y} C_{\mu] \sigma}{ }^{\sigma}+3 \partial_{y} A_{[\mu} \eta_{\nu] \rho} \\
& -\partial_{\rho} u_{\mu \nu}+\partial_{[\rho} u_{\mu \nu]}-2 \partial^{\sigma} u_{\sigma[\mu} \eta_{\nu] \rho}=\varphi_{\mu \nu, \rho} .
\end{aligned}
$$

\section{b. A-B duality}

Combining (B7) and (B8) gives

$\partial_{y} \partial^{\mu} u_{\mu \nu}=\frac{1}{2} \partial_{y}\left(\partial^{\mu} h_{\mu \nu}-\partial_{\nu} h_{\mu}{ }^{\mu}-\partial_{y} C_{\nu \mu}{ }^{\mu}+4 \partial_{y} A_{\nu}\right)-\partial^{\mu} \chi_{\mu \nu}$,

while the trace of $(\mathrm{B} 11)$ in $(\mu \rho)$ gives

$$
\partial^{\mu} u_{\mu \nu}=\frac{1}{2}\left(\partial^{\mu} h_{\mu \nu}-\partial_{\nu} h_{\mu}{ }^{\mu}-\partial_{y} C_{\nu \mu}{ }^{\mu}+4 \partial_{y} A_{\nu}\right)+\frac{1}{3} \varphi_{\mu \nu}{ }^{\mu} .
$$

Together, these two equations imply that locally, we can define a two-form $b$ such that

$$
\chi_{\mu \nu}=\frac{1}{2} \varepsilon_{\mu \nu \rho \sigma \tau} \partial^{\rho} b^{\sigma \tau}\left(x^{\mu}\right) .
$$

This two-form can be absorbed in $B$ (following exactly the same process as in Sec. A 2) such that Eq. (B8) reproduces (3.21).

\section{c. h-C duality}

Contracting (B11) with $\partial^{\mu}$, we can extract both symmetric and antisymmetric parts:

$$
\begin{aligned}
(\nu \rho) & : \mathcal{G}_{\nu \rho}+\frac{1}{2} \partial_{y}\left(\partial^{\mu} C_{\mu(\nu, \rho)}-\eta_{\nu \rho} \partial^{\mu} C_{\mu \sigma}{ }^{\sigma}+\partial_{(\rho} C_{\nu) \sigma}{ }^{\sigma}\right) \\
& =\partial^{\mu} \varphi_{\mu(\nu, \rho)},
\end{aligned}
$$




$$
[\nu \rho]:-\frac{1}{6} \varepsilon_{\lambda \sigma \tau \nu \rho} \partial^{\mu} \partial^{\lambda} C_{\mu}^{\sigma \tau}+\partial_{y}\left(\partial^{\mu} C_{\mu[\nu, \rho]}+\partial_{[\rho} C_{\nu] \sigma}{ }^{\sigma}-3 \partial_{[\rho} A_{\nu]}\right)+2 \partial^{\mu} \partial_{[\mu} u_{\nu \rho]}=2 \partial^{\mu} \varphi_{\mu[\nu, \rho]} .
$$

Using (B10) we can conclude that

$$
\partial^{\mu} \varphi_{\mu(\nu, \rho)}=0
$$

The divergence of (B9) reads

$$
-\frac{1}{6} \varepsilon_{\mu \nu \alpha \beta \gamma} \partial^{\rho} \partial^{\alpha} C_{\rho}^{\alpha \beta}+2 \partial^{\rho} \partial_{[\rho} u_{\mu \nu]}+\frac{1}{2} \partial_{y} \varepsilon_{\mu \nu \rho \alpha \beta} \partial^{\rho}\left(u^{\alpha \beta}-\frac{3}{2} B^{\alpha \beta}\right)=-\frac{1}{2} \partial^{\rho} \psi_{\mu \nu \rho},
$$

and combining it with (B16) and (B8), we eventually get

$$
2 \partial^{\mu} \varphi_{\mu[\nu, \rho]}=-\frac{1}{2} \partial^{\rho} \psi_{\mu \nu \rho}
$$

Together with (B17), one has

$$
\partial^{\mu}\left(2 \varphi_{\mu \nu, \rho}+\frac{1}{2} \partial^{\rho} \psi_{\mu \nu \rho}\right)=0
$$

such that locally there exist 5D tensors $c_{\mu \nu, \rho}$ and $a_{\mu \nu \rho}$, where $c$ is of $(2,1)$ type and $a$ is completely antisymmetric, such that

$$
2 \varphi_{\mu \nu, \rho}+\frac{1}{2} \partial^{\rho} \psi_{\mu \nu \rho}=\frac{1}{2} \varepsilon_{\mu \nu \alpha \beta \gamma} \partial^{\alpha}\left(c_{\rho}^{\beta \gamma}+a_{\rho}^{\beta \gamma}\right) .
$$

Consequently

$$
\begin{gathered}
\varphi_{\mu \nu}{ }^{\mu}=\frac{1}{4} \varepsilon_{\mu \nu \alpha \beta \gamma} \partial^{\alpha} a^{\beta \gamma \mu}, \\
\varphi_{\mu \nu, \rho}=\frac{1}{4} \varepsilon_{\mu \nu \alpha \beta \gamma} \partial^{\alpha}\left(c_{\rho}^{\beta \gamma}+a_{\rho}^{\beta \gamma}\right)-\frac{1}{4} \varepsilon_{\alpha \beta \gamma[\mu \nu} \partial^{\alpha}\left(c_{\rho]}^{\beta \gamma}+a_{\rho]}^{\beta \gamma}\right), \\
\psi_{\mu \nu \rho}=\varepsilon_{\alpha \beta \gamma[\mu \nu} \partial^{\alpha}\left(c^{\beta \gamma}{ }_{\rho]}+a_{\rho]}^{\beta \gamma}\right) .
\end{gathered}
$$

Plugging the expression for $\varphi$ and its trace back into (B11), one has

$$
\begin{aligned}
& 2 \partial_{[\mu} h_{\nu] \rho}+\frac{1}{2} \varepsilon_{\mu \nu \lambda \sigma \tau} \partial^{\lambda} C_{\rho}^{\sigma \tau}-\frac{1}{2} \varepsilon_{\lambda \sigma \tau[\mu \nu} \partial^{\lambda} C_{\rho]}^{\sigma \tau}+\partial_{y} C_{\mu \nu, \rho}-2 \partial_{y} A_{[\mu} \eta_{\nu] \rho}-2 \partial_{\rho} u_{\mu \nu}+2 \partial_{[\rho} u_{\mu \nu]} \\
& =\frac{1}{2} \varepsilon_{\mu \nu \alpha \beta \gamma} \partial_{\rho}^{\alpha}\left(c_{\rho}^{\beta \gamma}+a_{\rho}^{\beta \gamma}\right)-\frac{1}{2} \varepsilon_{\alpha \beta \gamma[\mu \nu} \partial^{\alpha}\left(c_{\rho]}^{\beta \gamma}+a_{\rho]}^{\beta \gamma}\right)+\frac{1}{3} \varepsilon_{\alpha \beta \gamma \sigma[\mu} \partial^{\alpha} a^{\beta \gamma \sigma} \eta_{\nu] \rho} .
\end{aligned}
$$

Then, using the following two Schouten identities

$$
\begin{gathered}
\varepsilon_{[\mu \nu}^{\alpha \beta \gamma} \partial_{\alpha} a_{\beta \gamma \rho]}=0=-\varepsilon^{\alpha \beta \gamma}{ }_{[\mu \nu} \partial_{\rho]} a_{\alpha \beta \gamma}+3 \partial_{\alpha} \varepsilon^{\alpha \beta \gamma}{ }_{[\mu \nu} a_{\rho] \beta \gamma}, \\
\varepsilon_{[\sigma \mu \alpha \beta \gamma} \partial^{\alpha} a^{\beta \gamma \sigma} \eta_{\nu] \rho}=0=2 \varepsilon_{\alpha \beta \gamma \sigma[\mu} \partial^{\alpha} a^{\beta \gamma \sigma} \eta_{\nu] \rho}-\partial_{\rho} \varepsilon_{\beta \gamma \sigma \mu \nu} a^{\beta \gamma \sigma}+3 \varepsilon_{\alpha \beta \gamma \mu \nu} \partial^{\alpha} a_{\rho}^{\beta \gamma},
\end{gathered}
$$

one obtains

$$
\begin{aligned}
& 2 \partial_{[\mu} h_{\nu] \rho}+\frac{1}{2} \varepsilon_{\mu \nu \lambda \sigma \tau} \partial^{\lambda}\left(C_{\rho}^{\sigma \tau}-c_{\rho}^{\sigma \tau}\right)+\left(H_{\mu \nu \rho}-\frac{1}{2} \varepsilon_{\mu \nu \rho \alpha \beta} F^{\alpha \beta}\right)+\partial_{y}\left(C_{\mu \nu, \rho}-2 A_{[\mu} \eta_{\nu] \rho}\right) \\
& -2 \partial_{\rho}\left(u_{\mu \nu}+\frac{1}{12} \varepsilon_{\mu \nu \alpha \beta \gamma} a^{\alpha \beta \gamma}\right)=0 .
\end{aligned}
$$

We recover the $6 \mathrm{D}$ equation (3.19) after the following redefinitions 


$$
\begin{aligned}
& u_{\mu \nu} \rightarrow u_{\mu \nu}+\frac{1}{12} \varepsilon_{\mu \nu \alpha \beta \gamma} a^{\alpha \beta \gamma}, \\
& B_{\mu \nu} \rightarrow B_{\mu \nu}-b_{\mu \nu}, \quad C_{\mu \nu, \rho} \rightarrow C_{\mu \nu, \rho}-c_{\mu \nu, \rho} .
\end{aligned}
$$

One can check that these redefinitions are consistent with the expression of $\psi$ in (B9). Finally, the derivatives of (3.19) and (3.21) give rise to the original 6D equations of motion (2.11) as discussed in Sec. III B above.

\section{The $\mathcal{N}=(4,0)$ model}

Here, we show how the second-order field equations obtained by variation of the Lagrangian (4.6) for the
$\mathcal{N}=(4,0)$ model can be integrated to the first-order field equations (3.34) and (3.36) which in turn imply the original 6D second-order self-duality equations (2.16).

\section{a. Field equations}

Let us first spell out the field equations obtained from variation of the Lagrangian (4.6). Apart from the Einstein equations (3.40) obtained by variation with respect to $h_{\mu \nu}$, all other field equations appear under total derivative $\partial_{y}$ and can be integrated into first-order equations up to functions that only depend on the five coordinates $x^{\mu}$. Explicitly, we may put them to the form

$$
\begin{aligned}
& \frac{1}{4} \varepsilon_{\alpha \beta \gamma[\mu \nu} \partial^{\alpha} C_{\rho]}^{\beta \gamma}-\partial_{[\rho} u_{\mu \nu]}+\frac{1}{2} \partial_{y} v_{[\rho, \mu \nu]}=\chi_{\mu \nu \rho}, \\
& \frac{1}{8} \varepsilon_{\alpha \beta \gamma[\mu \nu} \partial^{\alpha} C_{\rho]}^{\beta \gamma}+\frac{1}{4} \varepsilon_{\alpha \beta \gamma[\mu \nu} \partial^{\alpha} v_{\rho]}^{\beta \gamma}-\partial_{[\rho} u_{\mu \nu]}+\frac{1}{2} \partial_{y} v_{[\rho, \mu \nu]}=\psi_{\mu \nu \rho}, \\
& \partial_{[\mu} h_{\nu] \rho}+\partial^{\alpha} h_{\alpha[\mu} \eta_{\nu] \rho}-\eta_{\rho[\nu} \partial_{\mu]} h_{\alpha}{ }^{\alpha}+\frac{1}{4} \varepsilon_{\mu \nu \alpha \beta \gamma} \partial^{\alpha} C_{\rho}^{\beta \gamma}-\frac{1}{4} \varepsilon_{\alpha \beta \gamma[\mu \nu} \partial^{\alpha} C_{\rho]}^{\beta \gamma}-\partial_{\rho} u_{\mu \nu}+\partial_{[\rho} u_{\mu \nu]} \\
& -2 \partial^{\alpha} u_{\alpha[\mu} \eta_{\nu] \rho}+\frac{1}{2} \partial_{y}\left(C_{\mu \nu, \rho}+v_{\rho, \mu \nu}-v_{[\rho, \mu \nu]}\right)-\partial_{y} \eta_{\rho[\nu}\left(C_{\mu] \alpha}{ }^{\alpha}+v^{\alpha}{ }_{\mu] \alpha}\right)=\varphi_{\mu \nu, \rho} \\
& \partial_{[\mu} h_{\nu] \rho}+\partial^{\alpha} h_{\alpha[\mu} \eta_{\nu] \rho}-\eta_{\rho[\nu} \partial_{\mu]} h_{\alpha}{ }^{\alpha}+\frac{1}{8} \varepsilon_{\mu \nu \alpha \beta \gamma} \partial^{\alpha} C_{\rho}^{\beta \gamma}-\frac{1}{8} \varepsilon_{\alpha \beta \gamma[\mu \nu} \partial^{\alpha} C_{\rho]}^{\beta \gamma}-\partial_{\rho} u_{\mu \nu}+\partial_{[\rho} u_{\mu \nu]} \\
& -2 \partial^{\alpha} u_{\alpha[\mu} \eta_{\nu] \rho}+\frac{1}{2} \partial_{y}\left(C_{\mu \nu, \rho}+v_{\rho, \mu \nu}-v_{[\rho, \mu \nu]}\right)-\partial_{y} \eta_{\rho[\nu}\left(C_{\mu] \alpha}{ }^{\alpha}+v^{\alpha}{ }_{\mu] \alpha}\right)+\frac{1}{4} \varepsilon_{\mu \nu \alpha \beta \gamma} \partial^{\alpha} v_{\rho}^{\beta \gamma} \\
& -\frac{1}{4} \varepsilon_{\alpha \beta \gamma[\mu \nu} \partial^{\alpha} v_{\rho]}^{\beta \gamma}-\frac{1}{4} \partial^{\alpha} T_{\alpha \rho, \mu \nu}+\frac{1}{2} \partial_{[\mu} T_{\nu] \alpha, \rho}{ }^{\alpha}+\frac{1}{2} \eta_{\rho[\nu} \partial^{\alpha} T_{\mu] \beta, \alpha}{ }^{\beta}-\frac{1}{4} \eta_{\rho[\nu} \partial_{\mu]} T_{\alpha \beta}{ }^{\alpha \beta}=\theta_{\mu \nu, \rho}, \\
& \frac{1}{2} \varepsilon_{\alpha \beta \gamma \mu \nu} \partial^{\alpha} T_{\rho \sigma}^{\beta \gamma}+\frac{1}{2} \varepsilon_{\alpha \beta \gamma \rho \sigma} \partial^{\alpha} T^{\beta \gamma}{ }_{\mu \nu}-\varepsilon_{\alpha \beta \gamma[\mu \nu} \partial^{\alpha} T_{\rho \sigma]}^{\beta \gamma}+2 \partial_{(\mu}\left(C_{|\rho \sigma|, \nu)}-v_{\nu), \rho \sigma}\right) \\
& +2 \partial_{(\rho}\left(C_{|\mu \nu|, \sigma)}-v_{\sigma), \mu \nu}\right)-2 \partial^{\alpha}\left(C_{\alpha \rho,(\mu}-v_{(\mu,|\alpha \rho|}\right) \eta_{\nu) \sigma}-2 \partial^{\alpha}\left(C_{\alpha \sigma,(\mu}-v_{(\mu,|\alpha \sigma|}\right) \eta_{\nu) \rho} \\
& -2 \partial^{\alpha}\left(C_{\alpha \mu,(\rho}-v_{(\rho,|\alpha \mu|}\right) \eta_{\sigma) \nu}-2 \partial^{\alpha}\left(C_{\alpha \nu,(\rho}-v_{(\rho,|\alpha \nu|}\right) \eta_{\sigma) \mu}-2 \eta_{\sigma(\mu} \partial_{\nu)}\left(C_{\rho \alpha}{ }^{\alpha}-v_{\rho \alpha}^{\alpha}\right) \\
& -2 \eta_{\rho(\mu} \partial_{\nu)}\left(C_{\sigma \alpha}{ }^{\alpha}-v^{\alpha}{ }_{\sigma \alpha}\right)-2 \eta_{\mu(\rho} \partial_{\sigma)}\left(C_{\nu \alpha}{ }^{\alpha}-v_{\nu \alpha}^{\alpha}\right)-2 \eta_{\nu(\rho} \partial_{\sigma)}\left(C_{\mu \alpha}{ }^{\alpha}-v^{\alpha}{ }_{\mu \alpha}\right) \\
& +\partial_{y}\left(2 T_{\mu \nu, \rho \sigma}-4 \eta_{\sigma(\nu} T_{\mu) \alpha, \rho}{ }^{\alpha}-4 \eta_{\rho(\nu} T_{\mu) \alpha, \sigma}{ }^{\alpha}+2 \eta_{\mu(\rho} \eta_{\sigma) \nu} T_{\alpha \beta}^{\alpha \beta}\right) \\
& +4 \eta_{\mu(\rho} \eta_{\sigma) \nu} \partial^{\alpha}\left(C_{\alpha \beta}^{\beta}-v_{\alpha \beta}^{\beta}\right)=\phi_{\mu \nu, \rho \sigma} \text {. }
\end{aligned}
$$

Here, the functions $\chi_{\mu \nu \rho}$ and $\psi_{\mu \nu \rho}$ are totally antisymmetric, while $\varphi_{\mu \nu, \rho}$ and $\theta_{\mu \nu, \rho}$ are of $(2,1)$ type, and $\phi_{\mu \nu, \rho \sigma}$ is $(2,2)$. All of these functions do not depend on the sixth coordinate.

\section{b. h-C duality}

Using (3.40), one can show that the derivatives of (B30) and (B32) imply

$\partial^{\mu}\left(\varphi_{\mu \nu \rho}+\chi_{\mu \nu \rho}\right)=\frac{1}{2} \partial_{y}\left(\varepsilon_{\nu \rho \alpha \beta \gamma}\left(\chi^{\alpha \beta \gamma}-\psi^{\alpha \beta \gamma}\right)\right)=0$.
This implies that locally, we can define a totally antisymmetric $a_{\mu \nu \rho}$ and a $c_{\mu \nu, \rho}$ of $(2,1)$ type, such that

$$
\varphi_{\mu \nu \rho}+\chi_{\mu \nu \rho}=\frac{1}{4} \varepsilon_{\mu \nu \alpha \beta \gamma} \partial^{\alpha}\left(a_{\rho}^{\beta \gamma}+c_{\rho}^{\beta \gamma}\right) .
$$

Moreover

$$
\varphi_{\mu \nu}{ }^{\mu}=\frac{1}{4} \varepsilon_{\mu \nu \alpha \beta \gamma} a^{\beta \gamma \mu},
$$


thus

$$
\begin{aligned}
\partial_{[\mu} & h_{\nu] \rho}+\frac{1}{4} \varepsilon_{\mu \nu \alpha \beta \gamma} \partial^{\alpha} C_{\rho}^{\beta \gamma}-\partial_{\rho} u_{\mu \nu}+\frac{1}{2} \partial_{y}\left(C_{\mu \nu, \rho}+v_{\mu \nu, \rho}\right) \\
& =\varphi_{\mu \nu \rho}+\chi_{\mu \nu \rho}+\frac{2}{3} \varphi_{\alpha[\mu}{ }^{\alpha} \eta_{\nu] \rho} \\
& =\frac{1}{4} \varepsilon_{\mu \nu \alpha \beta \gamma} \partial^{\alpha} c_{\rho}^{\beta \gamma}+\frac{1}{12} \partial_{\rho} \varepsilon_{\mu \nu \alpha \beta \gamma} a^{\alpha \beta \gamma} .
\end{aligned}
$$

We thus recover the 6D equation (3.36) after redefining

$$
\begin{gathered}
C_{\mu \nu, \rho} \rightarrow C_{\mu \nu, \rho}-c_{\mu \nu, \rho} \\
u_{\mu \nu} \rightarrow u_{\mu \nu}+\frac{1}{12} \varepsilon_{\mu \nu \alpha \beta \gamma} a^{\alpha \beta \gamma} .
\end{gathered}
$$

\section{c. C-T duality}

Having established equation (3.36), we can hit it with another derivative to obtain the (modified) Curtright equation

$$
\begin{aligned}
& 3 \partial_{\tau} \partial^{[\tau} C_{\rho}^{\mu \nu]}-3 \partial_{\rho} \partial^{[\tau} C^{\mu \nu]} \\
& \quad=\frac{1}{2} \varepsilon^{\mu \nu \tau \kappa \lambda} \partial_{y}\left(\partial_{\tau} C_{\kappa \lambda, \rho}+\partial_{\tau} v_{\rho, \kappa \lambda}-\partial_{\rho} v_{\tau, \kappa \lambda}\right) .
\end{aligned}
$$

It remains to recover the duality equation (3.34). From (B30)-(B34), we obtain

$$
\mathcal{E}_{\mu \nu, \rho \sigma}=\Omega_{\mu \nu, \rho \sigma}(x),
$$

where

$\mathcal{E}_{\mu \nu, \rho \sigma} \equiv \frac{1}{2} \varepsilon_{\alpha \beta \gamma \mu \nu} \partial^{\alpha} T_{\rho \sigma}^{\beta \gamma}+2 \partial_{[\mu} C_{|\rho \sigma|, \nu]}-2 \partial_{[\rho} v_{\sigma], \mu \nu}+\partial_{y} T_{\mu \nu, \rho \sigma}$,

and $\Omega_{\mu \nu, \rho \sigma}$ is a combination of $\theta_{\mu \nu, \rho}$ and $\phi_{\mu \nu, \rho \sigma}$. For later use, we parametrize this field as

$$
\Omega_{\rho \sigma}^{\mu \nu}=\hat{\Omega}_{\rho \sigma}^{\mu \nu}+\frac{4}{3} \delta_{[\rho}^{[\mu} \Omega_{\sigma]}^{\nu]},
$$

with traceless $\hat{\Omega}_{\rho \sigma}^{\mu \nu}$ and the trace part given by

$$
{\Omega^{\mu \nu}}_{\rho \nu}=\Omega^{\mu}{ }_{\rho}+\frac{1}{3} \delta^{\mu}{ }_{\rho} \Omega_{\nu}^{\nu} .
$$

Equation (3.34) amounts to showing that

$$
\mathcal{E}_{\mu \nu, \rho \sigma}=0
$$

(after potential re-definition of $T_{\mu \nu, \rho \sigma}$ and $v_{\rho, \mu \nu}$ ). To begin with, using the field equations that are already established, we can show that the particular combination of derivatives

$$
\begin{aligned}
3 \partial_{[\mu} \Omega_{\rho \sigma]}^{\mu \nu} & =3 \partial^{\mu} \partial_{[\mu} C_{\rho \sigma]}^{\nu}-3 \partial^{\nu} \partial_{[\mu} C_{\rho \sigma]}{ }^{\mu}-3 \partial_{y} \partial_{[\mu} T_{\rho \sigma]}{ }^{\nu \mu} \\
& \stackrel{(B .42)}{=} 3 \partial^{\mu} \partial_{[\mu} C_{\rho \sigma]}{ }^{\nu}-3 \partial^{\nu} \partial_{[\mu} C_{\rho \sigma]}{ }^{\mu} \\
& -\frac{1}{2} \varepsilon^{\mu \kappa \lambda \rho \sigma} \partial_{y}\left(\partial_{\mu} C_{\kappa \lambda}{ }^{\nu}+\partial_{\lambda} v^{\nu}{ }_{\mu \kappa}-\partial^{\nu} v_{\lambda, \mu \kappa}\right) \\
& \stackrel{(B .41)}{=} 0
\end{aligned}
$$

vanishes. In particular, contraction of this equation shows that

$2 \partial_{\mu} \Omega_{\rho \nu}^{\mu \nu}-\partial_{\rho} \Omega^{\mu \nu}{ }_{\mu \nu}=0 \quad \Rightarrow \quad \partial_{\mu}\left({\Omega^{\mu}}_{\rho}-\delta^{\mu}{ }_{\rho} \Omega_{\nu}^{\nu}\right)=0$.

This can be integrated to

$\Omega^{\mu}{ }_{\rho}-\delta^{\mu}{ }_{\rho} \Omega^{\nu}{ }_{\nu}=\partial_{\lambda} \omega^{\lambda \mu}{ }_{\rho} \Leftrightarrow \Omega^{\mu}{ }_{\rho}=\partial_{\lambda} \omega^{\lambda \mu}{ }_{\rho}-\frac{1}{4} \delta^{\mu}{ }_{\rho} \partial_{\lambda} \omega^{\lambda \kappa}{ }_{\kappa}$.

Next, we observe that (B47) gives rise to the secondorder differential equation

$$
0=\partial_{\mu} \partial_{[\tau} \Omega_{\rho \sigma]}^{\mu \nu} .
$$

Using the parametrization (B44) and the relation (B48), this equation reduces to

$$
0=\partial_{\mu} \partial_{[\tau} \hat{\Omega}_{\rho \sigma]}^{\mu \nu},
$$

for the traceless part of $\Omega$. Dualizing the first two indices on $\hat{\Omega}$, defines the object

$$
\hat{\Omega}_{\alpha \beta \gamma, \rho \sigma} \equiv \frac{1}{2} \varepsilon_{\alpha \beta \gamma \mu \nu} \hat{\Omega}_{\rho \sigma}^{\mu \nu},
$$

corresponding to an irreducible Young tableau $\square$. Using the generalized Poincaré Lemma [30] for this object then allows to integrate up equation (B51) into

$\hat{\Omega}_{\rho \sigma}^{\mu \nu}=\frac{1}{2} \varepsilon^{\mu \nu \alpha \beta \gamma} \partial_{\alpha} t_{\beta \gamma, \rho \sigma}+2 \partial_{[\rho} s_{\sigma \nu}^{\mu \nu}+\frac{4}{3} \delta_{[\rho \mid}^{[\mu} \partial_{\kappa} s^{\nu] \kappa}{ }_{\mid \sigma]}$,

in terms of two gauge parameters $t_{\mu \nu, \rho \sigma}$ and $s_{\mu \nu, \rho}$. The first one corresponds to an irreducible Young tableau $\square$, while the parameter $s_{\mu \nu, \rho}$ is antisymmetric in its first two indices and traceless $s_{\mu \nu}{ }^{\nu}=0$, - such that its dual $\hat{s}_{\alpha \beta \gamma, \rho}=$ $\frac{1}{2} \varepsilon_{\alpha \beta \gamma \mu \nu} s^{\mu \nu}{ }_{\rho}$ corresponds to an irreducible Young tableau . The last term in (B53) implements the projection onto the traceless part.

Putting together (B53) and (B49), we arrive at

$$
\begin{aligned}
\Omega_{\rho \sigma}^{\mu \nu}= & \left.\frac{1}{2} \varepsilon^{\mu \nu \alpha \beta \gamma} \partial_{\alpha} t_{\beta \gamma, \rho \sigma}+2 \partial_{[\rho} s_{\sigma]}^{\mu \nu}+\frac{4}{3} \delta_{[\rho \mid}^{[\mu} \partial_{\kappa} s^{\nu] \kappa} \mid \sigma\right] \\
& -\frac{4}{3} \delta_{[\rho \mid}\left[\mu \partial_{\kappa} \omega^{\nu] \kappa}{ }_{\mid \sigma]}-\frac{1}{3} \delta^{\mu \nu}{ }_{\rho \sigma} \partial_{\lambda} \omega^{\lambda \kappa}{ }_{\kappa} .\right.
\end{aligned}
$$


We can plug this back into (B47) to arrive at

$$
\begin{aligned}
0= & 3 \partial_{[\mu} \Omega^{\mu \nu}{ }_{\rho \sigma]}=\frac{4}{3} \partial_{\mu} \partial_{[\rho} s^{\mu \nu}{ }_{\sigma]}-\frac{4}{3} \partial_{\mu} \partial_{[\rho} \omega^{\mu \nu}{ }_{\sigma]} \\
& +\frac{1}{3} \delta_{[\sigma}{ }^{\nu} \partial_{\rho]} \partial_{\lambda} \omega^{\lambda \kappa}{ }_{\kappa},
\end{aligned}
$$

which in turn is a curl in $[\rho \sigma]$ and can be integrated into

$$
\partial_{\mu} s^{\mu \nu}{ }_{\sigma}-\partial_{\mu} \omega^{\mu \nu}{ }_{\sigma}+\frac{1}{4} \delta_{\sigma}{ }^{\nu} \partial_{\lambda} \omega^{\lambda \kappa}{ }_{\kappa}=\partial_{\sigma} \xi^{\nu}
$$

This further reduces the result (B54) and allows to put it into the form
$\Omega_{\rho \sigma}^{\mu \nu}=\frac{1}{2} \varepsilon^{\mu \nu \alpha \beta \gamma} \partial_{\alpha} t_{\beta \gamma, \rho \sigma}+2 \partial_{[\rho}\left(s^{\mu \nu}{ }_{\sigma]}+\frac{2}{3} \delta_{\sigma]}^{\left[\mu \xi^{\nu]}\right.}\right)$.

Using this result in (B42), we finally arrive at

$$
\mathcal{E}_{\mu \nu, \rho \sigma}=0
$$

upon shifting

$T_{\mu \nu, \rho \sigma} \rightarrow T_{\mu \nu, \rho \sigma}+t_{\mu \nu, \rho \sigma}, \quad v_{\rho, \mu \nu} \rightarrow v_{\rho, \mu \nu}+s_{\mu \nu, \rho}+\frac{2}{3} \eta_{\rho[\mu} \xi_{\nu]}$.

We have thus shown that also the Eq. (3.34) follows from the Lagrangian (4.6). Finally, derivatives of (3.34) and (3.36) give rise to the original $6 \mathrm{D}$ equations of motion (2.16) as discussed in Sec. III C above.
[1] C. Hull, Strongly coupled gravity and duality, Nucl. Phys. B583, 237 (2000).

[2] C. M. Hull, Symmetries and compactifications of $(4,0)$ conformal gravity, J. High Energy Phys. 12 (2000) 007.

[3] M. Chiodaroli, M. Günaydin, and R. Roiban, Superconformal symmetry and maximal supergravity in various dimensions, J. High Energy Phys. 03 (2012) 093.

[4] A. Anastasiou, L. Borsten, M. Duff, L. Hughes, and S. Nagy, A magic pyramid of supergravities, J. High Energy Phys. 04 (2014) 178.

[5] L. Borsten, $D=6, \mathcal{N}=(2,0)$ and $\mathcal{N}=(4,0)$ theories, Phys. Rev. D 97, 066014 (2018).

[6] J. A. Strathdee, Extended Poincaré supersymmetry, Int. J. Mod. Phys. A 02, 273 (1987).

[7] C. Hull, BPS supermultiplets in five-dimensions, J. High Energy Phys. 06 (2000) 019.

[8] D. S. Berman and M. J. Perry, Generalized geometry and M theory, J. High Energy Phys. 06 (2011) 074.

[9] A. Coimbra, C. Strickland-Constable, and D. Waldram, $E_{d(d)} \times \mathbb{R}^{+}$generalised geometry, connections and $\mathrm{M}$ theory, J. High Energy Phys. 02 (2014) 054.

[10] O. Hohm and H. Samtleben, Exceptional Form of $D=11$ Supergravity, Phys. Rev. Lett. 111, 231601 (2013).

[11] O. Hohm and H. Samtleben, Exceptional field theory I: $E_{6(6)}$ covariant form of M-theory and type IIB, Phys. Rev. D 89, 066016 (2014).

[12] A. Baguet, O. Hohm, and H. Samtleben, $E_{6(6)}$ exceptional field theory: Review and embedding of type IIB, Proc. Sci., CORFU2014 (2015) 133.

[13] M. Henneaux and C. Teitelboim, Dynamics of chiral (selfdual) p-forms, Phys. Lett. B 206, 650 (1988).

[14] M. Henneaux, V. Lekeu, and A. Leonard, Chiral tensors of mixed Young symmetry, Phys. Rev. D 95, 084040 (2017).

[15] M. Henneaux, V. Lekeu, and A. Leonard, The action of the (free) (4, 0)-theory, J. High Energy Phys. 01 (2018) 114; Erratum, 05 (2018) 105.
[16] M. Henneaux, V. Lekeu, J. Matulich, and S. Prohazka, The action of the (free) $\mathcal{N}=(3,1)$ theory in six spacetime dimensions, J. High Energy Phys. 06 (2018) 057.

[17] M. Henneaux and C. Teitelboim, Duality in linearized gravity, Phys. Rev. D 71, 024018 (2005).

[18] Y. Tanii, $N=8$ supergravity in six dimensions, Phys. Lett. 145B, 197 (1984).

[19] T. Curtright, Generalized gauge fields, Phys. Lett. 165B, 304 (1985).

[20] P. Pasti, D. P. Sorokin, and M. Tonin, On Lorentz invariant actions for chiral $p$-forms, Phys. Rev. D 55, 6292 (1997).

[21] A. Sen, Self-dual forms: Action, Hamiltonian and compactification, J. Phys. A 53, 084002 (2020).

[22] K. Mkrtchyan, On covariant actions for chiral $p$-forms, J. High Energy Phys. 12 (2019) 076.

[23] P. C. West, $E_{11}$ and $M$ theory, Classical Quantum Gravity 18, 4443 (2001).

[24] C. Hull, Duality in gravity and higher spin gauge fields, J. High Energy Phys. 09 (2001) 027.

[25] E. Cremmer, Supergravities in 5 dimensions, in Superspace and Supergravity: Proceedings Nuffield Gravity Workshop, edited by S. Hawking and M. Rocek (Cambridge University Press, Cambridge, England, 1980).

[26] X. Bekaert and M. Henneaux, Comments on chiral $p$ forms, Int. J. Theor. Phys. 38, 1161 (1999).

[27] M. Henneaux, V. Lekeu, and A. Leonard, A note on the double dual graviton, J. Phys. A 53, 014002 (2020).

[28] R. Bonezzi and O. Hohm, Duality hierarchies and differential graded Lie algebras, arXiv:1910.10399.

[29] R. Minasian, C. Strickland-Constable, and Y. Zhang, On symmetries and dynamics of exotic supermultiplets, arXiv: 2007.08888.

[30] X. Bekaert and N. Boulanger, Tensor gauge fields in arbitrary representations of $G L(D, \mathbb{R})$ : Duality and Poincaré lemma, Commun. Math. Phys. 245, 27 (2004). 